\title{
Mathematical analysis on an age-structured SIS epidemic model with nonlocal diffusion
}

\author{
Hao Kang ${ }^{1}$ · Shigui Ruan ${ }^{1}$
}

Received: 4 August 2020 / Revised: 2 June 2021 / Accepted: 13 June 2021 / Published online: 26 June 2021 (c) The Author(s), under exclusive licence to Springer-Verlag GmbH Germany, part of Springer Nature 2021

\begin{abstract}
In this paper we propose an age-structured susceptible-infectious-susceptible epidemic model with nonlocal (convolution) diffusion to describe the geographic spread of infectious diseases via long-distance travel. We analyze the well-posedness of the model, investigate the existence and uniqueness of the nontrivial steady state corresponding to an endemic state, and study the local and global stability of this nontrivial steady state. Moreover, we discuss the asymptotic properties of the principal eigenvalue and nontrivial steady state with respect to the nonlocal diffusion rate. The analysis is carried out by using the theory of semigroups and the method of monotone and positive operators. The spectral radius of a positive linear operator associated to the solution flow of the model is identified as a threshold.
\end{abstract}

Keywords Age-structure - Nonlocal diffusion - SIS model - Semigroup theory · Principal eigenvalue $\cdot$ Monotone and positive operators $\cdot$ Global dynamics

Mathematics Subject Classification 35L60 · 92D25 · 47H07

\section{Introduction}

Age structure and spatial movement are two important features on the geographic spread of infectious diseases. The age could be chronological age (the age of the host), infection age (the time elapsed since infection), recovery age (the time elapsed since the last infection), class age (the length of time in the present group), etc. After the early work of Webb (1980, 1982), various age-structured epidemic models with spatial diffusion described by combined hyperbolic-parabolic partial differential equa-

Research was partially supported by National Science Foundation (DMS-1853622).

\footnotetext{
$凶 \quad$ Shigui Ruan

ruan@math.miami.edu

1 Department of Mathematics, University of Miami, Coral Gables, FL 33146, USA
} 
tions have been proposed and studied, see Chekroun and Kuniya (2020), Di Blasio (2010), Fitzgibbon et al. (1994, 1996), Kim (2006), Kubo and Langlais (1994), Kuniya et al. (2018), Kuniya and Oizumi (2015), Chekroun and Kuniya (2020), Langlais and Busenberg (1997), and the references cited therein. In these studies, the classical Laplace diffusion was used to describe the random walk of the host in a connected domain. However, in modern time many infectious diseases are spread geographically via long-distance travel such as air travel (Findlater and Bogoch (2018)). It seems that the classical Laplace diffusion is unsuitable to model the spatial spread of infectious diseases via long-distance travel (Blyuss (2005) and Ruan (2007)).

The nonlocal (convolution) diffusion provides a way to describe long-distance dispersal (Yang et al. (2013) and $\mathrm{Xu}$ et al. (2020)). The goal of this paper is to propose a new type of epidemic models, namely age-structured susceptible-infectioussusceptible (SIS) epidemic model with nonlocal spatial diffusion. Note that for scalar linear and nonlinear age-structured equations with nonlocal diffusion, recently we (Kang et al. (2020b), Kang and Ruan (2021b) and two forthcoming papers) developed some basic theories including the semigroup of linear operators, asymptotic behavior, spectral theory, asynchronous exponential growth, strong maximum principle, global dynamics, etc. Here we generalize our previous modeling scheme and techniques to study the dynamics of an age-structured SIS epidemic model with nonlocal spatial diffusion.

Let $S(t, a, x)$ and $I(t, a, x)$ be the densities of susceptible and infective individuals of age $a \geq 0$ at time $t \geq 0$ in position $x \in \Omega \subset \mathbb{R}^{N}$, respectively, where $\Omega$ is a bounded subset of $\mathbb{R}^{N}$ and $a<\bar{a}^{+}$, the maximum age which is finite. Let $\lambda(t, a, x)$ be the force of infection of infectious individuals to susceptible individuals of age $a$ at time $t$ in position $x$, and $\gamma(a, x)$ be the recovery rate of infective individuals of age $a$ in position $x$. We consider the following system of age-structured equations with nonlocal spatial diffusion

$$
\begin{cases}\left(\partial_{t}+\partial_{a}\right) S(t, a, x)=d[\mathcal{L} S](t, a, x)-\lambda(t, a, x) S(t, a, x)- & \mu(a, x) S(t, a, x)+\gamma(a, x) I(t, a, x), \\ & t>0, a \in\left(0, a^{+}\right], x \in \bar{\Omega}, \\ \left(\partial_{t}+\partial_{a}\right) I(t, a, x)=d[\mathcal{L} I](t, a, x)+\lambda(t, a, x) S(t, a, x)- & (\mu(a, x)+\gamma(a, x)) I(t, a, x), \\ & t>0, a \in\left(0, a^{+}\right], x \in \bar{\Omega}, \\ S(t, 0, x)=P(t, 0, x), \quad I(t, 0, x)=0, & t>0, x \in \bar{\Omega}, \\ S(0, a, x)=S_{0}(a, x), \quad I(0, a, x)=I_{0}(a, x), & a \in\left[0, a^{+}\right], x \in \bar{\Omega},\end{cases}
$$

where $\mu(a, x)$ denotes the mortality of individuals of age $a$ in position $x$ and the nonlocal operator $\mathcal{L}$ is defined by

$$
[\mathcal{L} u](t, a, x):=(J * u-u)(t, a, x)=\int_{\Omega} J(x-y) u(t, a, y) d y-u(t, a, x) .
$$

$P(t, 0, x)$ is the boundary condition given by

$$
P(t, 0, x)=\int_{0}^{a^{+}} \int_{\Omega} \beta(a, x, y) P(t, a, y) d y d a, t>0, x \in \bar{\Omega},
$$


in which $\beta(a, x, y)$ denotes the birth rate at which individuals of age $a$ in position $y$ give to newborns in position $x$ and $P(t, a, x)=S(t, a, x)+I(t, a, x)$ represents the total population. $S_{0}(a, x)$ and $I_{0}(a, x)$ are positive initial value functions. We mention that the nonlocal diffusion operator in (1.1) corresponds to an elliptic operator with zero Dirichlet boundary condition. The convolution $\int_{\Omega} J(x-y) u(t, a, y) d y$ is the rate at which individuals are arriving at position $x$ from other places while $\int_{\Omega} J(y-x) u(t, a, x) d y$ is the rate at which individuals are leaving location $x$ for other locations. Moreover, $J$ is a diffusion kernel which is a $C^{0}$ nonnegative function with unit integral representing the spatial dispersal and $J(0)>0$; i.e.,

$$
\int_{\mathbb{R}^{N}} J(x) d x=1, \quad J(x) \geq 0, \quad \forall x \in \mathbb{R}^{N}
$$

In addition, we assume that the force of infection $\lambda(t, a, x)$ is given by

$$
\lambda(t, a, x)=\int_{0}^{a^{+}} \int_{\Omega} k(a, \sigma, x, y) I(t, \sigma, y) d y d \sigma,
$$

where $k(a, \sigma, x, y)$ denotes the rate of disease transmission from infective individuals of age $\sigma$ in position $y$ to susceptible individuals of age $a$ in position $x$.

(a) Age-structured SIS epidemic models. If $S(t, a, x)=S(t, a)$ and $I(t, a, x)=$ $I(t, a)$ do not depend on the spatial variable, then model (1.1) reduces to the following age-structured SIS epidemic model provided $\int_{\Omega} J(x-y) d y=1$ for all $x \in \Omega$ :

$$
\left\{\begin{array}{l}
\left(\partial_{t}+\partial_{a}\right) S(t, a)=-S(t, a) \int_{0}^{a^{+}} k(a, \sigma) I(t, \sigma) d \sigma-\mu(a) S(t, a)+\gamma(a) I(t, a), t>0, a \in\left(0, a^{+}\right], \\
\left(\partial_{t}+\partial_{a}\right) I(t, a)=S(t, a) \int_{0}^{a^{+}} k(a, \sigma) I(t, \sigma) d \sigma-(\mu(a)+\gamma(a)) I(t, a), t>0, a \in\left(0, a^{+}\right], \\
S(t, 0)=P(t, 0), \quad I(t, 0)=0, t>0, \\
S(0, a)=S_{0}(a), \quad I(0, a)=I_{0}(a), a \in\left[0, a^{+}\right] .
\end{array}\right.
$$

Such age-structured epidemic models have been studied extensively in the literature, see Busenberg et al. (1991), Iannelli (1995), Iannelli et al. (1999), Iannelli et al. (1992), Iannelli and Milner (2017), Inaba (1990, 2014, 2017), Kang et al. (2020a), Kuniya and Inaba (2013), Kuniya (2014), Kuniya and Iannelli (2014), Kuniya et al. (2016, 2018), Thieme (1991), and Webb (1984). Since the model is independent of the location, most studies focused on the temporal dynamics of these models.

(b) Nonlocal SIS epidemic models. If $S(t, a, x)=S(t, x)$ and $I(t, a, x)=I(t, x)$ do not depend on the age variable, then model (1.1) becomes the following nonlocal SIS epidemic model:

$$
\left\{\begin{aligned}
\partial_{t} S(t, x)= & d\left[\int_{\Omega} J(x-y) S(t, y) d y-S(t, x)\right]-S(t, x) \int_{\Omega} k(x, y) I(t, y) d y \\
& -\mu(x) S(t, x)+\gamma(x) I(t, x), t>0, x \in \bar{\Omega}, \\
\partial_{t} I(t, x)= & d\left[\int_{\Omega} J(x-y) I(t, y) d y-I(t, x)\right]+S(t, x) \int_{\Omega} k(x, y) I(t, y) d y \\
& -(\mu(x)+\gamma(x)) I(t, x), t>0, x \in \bar{\Omega} \\
S(0, x)= & S_{0}(x), \quad I(0, x)=I_{0}(a, x), x \in \bar{\Omega} .
\end{aligned}\right.
$$


Nonlocal dispersal epidemic models (without age structure) including (1.5) have also been studied by some researchers, see Xu et al. (2020), Yang and Li (2017), and Yang et al. (2013, 2019). These studies were mainly concerned with the existence of traveling wave solutions in such nonlocal dispersal models. For existence and stability of steady states in such models, see Yang et al. (2019) and Zhao and Ruan (2018). Notice that model (1.5) is more general than those considered in $\mathrm{Xu}$ et al. (2020), Yang and Li (2017), and Yang et al. (2013, 2019), since it includes not only nonlocal dispersal terms but also nonlocal interaction terms.

(c) Age-structured SIS epidemic models with Laplace diffusion. If the spatial domain $\Omega \subset \mathbb{R}^{N}$ is connected and the dispersal of individuals follows is a random walk, we obtain the following age-structured SIS epidemic model with Laplace diffusion:

$$
\begin{cases}\left(\partial_{t}+\partial_{a}\right) S(t, a, x)=d \Delta S(t, a, x)-\lambda(t, a, x) S(t, a, x)- & \mu(a, x) S(t, a, x)+\gamma(a, x) I(t, a, x), \\ & t>0, a \in\left(0, a^{+}\right], x \in \Omega, \\ \left(\partial_{t}+\partial_{a}\right) I(t, a, x)=d \Delta I(t, a, x)+\lambda(t, a, x) S(t, a, x)- & (\mu(a, x)+\gamma(a, x)) I(t, a, x), \\ & t>0, a \in\left(0, a^{+}\right], x \in \Omega, \\ S(t, 0, x)=P(t, 0, x), \quad I(t, 0, x)=0, & t>0, x \in \Omega, \\ S(0, a, x)=S_{0}(a, x), \quad I(0, a, x)=I_{0}(a, x), & a \in\left[0, a^{+}\right], x \in \Omega,\end{cases}
$$

where $\lambda(t, a, x)$ is given by (1.3). There could be appropriate boundary conditions on $\partial \Omega$ in (1.6), but we omit them. Various age-structured epidemic models with Laplace diffusion related to model (1.6) have been proposed and studied, see Di Blasio (2010), Kim (2006), Kubo and Langlais (1994), Kuniya et al. (2018), Chekroun and Kuniya (2020), Kuniya and Oizumi (2015), Langlais and Busenberg (1997), and the references cited therein. However, due to their complex structures, the spatial-temporal dynamics of this kind of epidemic models are not well-understood.

Here we provide a comprehensive analysis on the dynamics of age-structured SIS epidemic model (1.1) with nonlocal diffusion. We first analyze the well-posedness of the model, then investigate the existence and uniqueness of the nontrivial steady state, and finally study the local and global stability of this nontrivial steady state. Moreover, we consider the asymptotic properties of the principal eigenvalue and nontrivial steady state with respect to the diffusion rate. We would like to mention that the analysis carried out in this paper is also valid for the age-structured SIS epidemic model (1.6) with random diffusion. We also refer to our recent study in Kang and Ruan (2021a) on the approximation of random diffusion by nonlocal diffusion with properly rescaled kernels in age-structured models.

The paper is organized as follows. In Sect. 2, we define the function spaces, introduce a key lemma and two theorems, and make some assumptions. In Sect. 3, we study the well-posedness of the model and provide an important monotone property of the semigroup generated by the solution flow. In Sect. 4, we investigate the existence of the nontrivial steady state. In Sect. 5 we define the basic reproduction number. A result on the uniqueness of the nontrivial steady state is obtained in Sect. 6. Then, we study local stability of the nontrivial steady state and asymptotic properties of the principal eigenvalue and nontrivial steady state in terms of the diffusion rate $d$ in Sect. 7. In Sect. 8, we analyze global stability of the nontrivial steady state. We end up the paper with a discussion in Sect. 9. 


\section{Preliminaries}

Define the function spaces as follows:

$$
Z:=L^{1}(\Omega), \quad X:=L^{1}\left(\left(0, a^{+}\right), Z\right)=L^{1}\left(\left(0, a^{+}\right) \times \Omega\right)
$$

with positive cones

$$
\begin{aligned}
& Z_{+}:=L_{+}^{1}(\Omega):=\{f \in Z: f \geq 0, \text { a.e. }\} \\
& X_{+}:=L_{+}^{1}\left(\left(0, a^{+}\right), Z\right):=\{f \in X: f \geq 0, \text { a.e. }\}
\end{aligned}
$$

respectively, Then we give a lemma based on Guo and Chan (1994), and its proof can be found in (Kang et al. 2020b, Lemma 3.1).

Lemma 2.1 Assume that $f \in L_{+}^{\infty}\left(\left(0, a^{+}\right) \times \Omega\right)$, then there exists a unique mild solution $u(a, x), 0 \leq \tau \leq a \leq a^{+}$, to the evolution equation on $X$ for any initial data $\phi \in Z$

$$
\begin{cases}\partial_{a} u(a, x)=[-f(a, x)+d \mathcal{L}] u(a, x), & a \in\left(0, a^{+}\right), x \in \bar{\Omega}, \\ u(\tau, x)=\phi(x), & x \in \bar{\Omega} .\end{cases}
$$

Here a mild solution means that $u \in C\left(\left[0, a^{+}\right], Z\right)$. Define the solution operator of the initial value problem (2.1) by

$$
(\mathcal{F}(\tau, a) \phi)(x)=u(a, x), \quad \forall \phi \in Z \text {. }
$$

Then $\{\mathcal{F}(\tau, a)\}_{0 \leq \tau \leq a \leq a^{+}}$is a family of linear uniformly bounded positive operators on $Z$ and is strongly continuous in $\tau$ and $a$. Furthermore,

$$
e^{-\int_{\tau}^{a} \bar{f}(\rho) d \rho} e^{d \mathcal{L}(a-\tau)} \leq \mathcal{F}(\tau, a) \leq e^{-\int_{\tau}^{a} \underline{f}(\rho) d \rho} e^{d \mathcal{L}(a-\tau)},
$$

where $\underline{f}(a):=\inf _{x \in \Omega} f(a, x)$ and $\bar{f}(a):=\sup _{x \in \Omega} f(a, x)$.

We make the following assumptions on each parameter.

\section{Assumption 2.2}

(i) $\gamma$ and $\mu$ are belonging to $L_{+}^{\infty}\left(\left(0, a^{+}\right) \times \Omega\right)$ with $\gamma^{+}:=\|\gamma\|_{L_{+}^{\infty}\left(\left(0, a^{+}\right) \times \Omega\right)}$ and $\mu^{+}:=\|\mu\|_{L_{+}^{\infty}\left(\left(0, a^{+}\right) \times \Omega\right)}$

(ii) $k(a, \sigma, x, y)=0$ for $x, y \in \mathbb{R}^{N} \backslash \Omega$ or for $a, \sigma<0$ and $a, \sigma>a^{+}$;

(iii) $k(a, \sigma, x, y)$ satisfies

$$
\lim _{\|(\kappa, h)\| \rightarrow 0} \int_{0}^{a^{+}} \int_{\Omega}|k(a+\kappa, \sigma, x+h, y)-k(a, \sigma, x, y)| d x d a=0
$$

uniformly for $\sigma \in\left[0, a^{+}\right]$and $y \in \Omega$, where $\kappa \in \mathbb{R}, h \in \mathbb{R}^{N}$ and $\|\cdot\|$ denotes the usual Euclidean norm in $\mathbb{R}^{N+1}$; 
(iv) There exists two positive functions $k_{1} \in L_{+}^{1}\left(\left(0, a^{+}\right) \times \Omega\right)$ and $k_{2} \in L_{+}^{\infty}\left(\left(0, a^{+}\right) \times\right.$ $\Omega$ ) respectively such that

$$
\epsilon k_{1}(a, x) k_{2}(\sigma, y) \leq k(a, \sigma, x, y) \leq k_{1}(a, x) k_{2}(\sigma, y)
$$

for all $(a, x),(\sigma, y) \in \mathbb{R}_{+} \times \bar{\Omega}$, where $\epsilon \in(0,1)$;

(v) $k_{1}(a, x)$ has positive upper and lower bounds $k^{+}$and $k^{-}$such that $k^{+} \geq k_{1}(a, x) \geq$ $k^{-}>0$ for all $(a, x) \in\left[0, a^{+}\right] \times \bar{\Omega}$.

The lower bound in Assumption 2.2-(v) means that the transmission can occur between every pair of susceptible and infectious individuals from an epidemiological point of view.

Next we recall some basic concepts of nonsupporting operators and the fixed point theorem from Inaba (1990) (see also (Inaba 2014, Proposition 7.7)). Let $B(Y)$ be the set of bounded linear operators from $Y$ to $Y$, where $Y$ is a Banach space with a positive cone $Y_{+} . L \in B(Y)$ is said to be positive if $L\left(Y_{+}\right) \subset Y_{+} . L \in B(Y)$ is said to be strongly positive if $\langle f, L \psi\rangle>0$ for every pair $\psi \in Y_{+} \backslash\{0\}, f \in Y_{+}^{*} \backslash\{0\}$, where $Y_{+}^{*}$ is the space of positive linear functionals on $Y$. For $L, V \in B(Y)$, we say $L \geq V$ if $(L-V)\left(Y_{+}\right) \subset Y_{+}$. A positive operator $L \in B(Y)$ is said to be nonsupporting if for every pair $\psi \in Y_{+} \backslash\{0\}$ and $f \in Y_{+}^{*} \backslash\{0\}$, there exists a positive integer $p=p(\psi, f)$ such that $\left\langle f, L^{n} \psi\right\rangle>0$ for all $n \geq p$. $r(L)$ denotes the spectral radius of $L \in B(Y)$, $\sigma(L)$ denotes the spectrum of $L$, and $\sigma_{P}(L)$ denotes the point spectrum of $L$.

Theorem 2.3 (Inaba (1990)) Let $Y$ be a real Banach space and $Y_{+}$be its positive cone. Let $\Psi$ be a positive operator from $E_{+}$to itself and $L:=\Psi^{\prime}[0]$ be its Fréchet derivative at 0 . If

(i) $\Psi(0)=0$;

(ii) $\Psi$ is compact and bounded;

(iii) L has a positive eigenvector $v_{0} \in Y_{+} \backslash\{0\}$ associated with an eigenvalue $\lambda_{0}>1$;

(iv) L has no eigenvector in $Y_{+}$associated with the eigenvalue 1 ,

then $\Psi$ has at least one nontrivial fixed point in $Y_{+}$.

In the case where $L$ is a majorant of $\Psi$ (that is, $L$ is a linear operator such that $\Psi(\phi) \leq L \phi$ for any $\phi \in Y_{+}$), the following theorem also holds (see (Inaba 2014, Proposition 7.8)).

Theorem 2.4 (Inaba (2014)) Let $Y$ be a real Banach space and $Y_{+}$be its positive cone. Let $\Psi$ be a positive operator from $Y_{+}$to itself and $L$ be its compact and nonsupporting majorant. Then, $\Psi$ has no trivial fixed point in $Y_{+}$provided $r(L) \leq 1$.

\section{Well-posedness}

\subsection{McKendrick equation with nonlocal diffusion}

Let $P(t, a, x)$ be the total population density at time $t \geq 0$ with age $a \geq 0$ in position $x \in \Omega$. Then the total population $P$ is governed by the McKendrick equation with 
nonlocal diffusion (see Kuniya and Oizumi (2015)):

$$
\begin{cases}\left(\partial_{t}+\partial_{a}\right) P(t, a, x)=d[\mathcal{L} P](t, a, x)-\mu(a, x) P(t, a, x), & t>0, a \in\left(0, a^{+}\right], x \in \bar{\Omega}, \\ P(t, 0, x)=\int_{0}^{a^{+}} \int_{\Omega} \beta(a, x, y) P(t, a, y) d y d a, & t>0, x \in \bar{\Omega}, \\ P(0, a, x)=P_{0}(a, x) \in X_{+}, & a \in\left[0, a^{+}\right], x \in \bar{\Omega},\end{cases}
$$

where $\mu(a, x)$ represents the mortality of an individual of age $a$ in position $x, \beta(a, x, y)$ denotes the birth rate at which an individual of age $a$ in position $y$ gives to a newborn in position $x$. We make the following assumptions on these parameters.

\section{Assumption 3.1}

(i) $\mu$ is belonging to $L_{+}^{\infty}\left(\left(0, a^{+}\right) \times \Omega\right)$ with $\mu^{+}:=\|\mu\|_{L_{+}^{\infty}\left(\left(0, a^{+}\right) \times \Omega\right)}$;

(ii) $\beta(a, \cdot, \cdot)=0$ for $a<0$ and $a>a^{+}$with

$$
\lim _{\|h\| \rightarrow 0} \int_{\Omega}|\beta(a, x+h, y)-\beta(a, x, y)| d x=0
$$

uniformly in $a \in\left(0, a^{+}\right), y \in \bar{\Omega}$, where $h \in \mathbb{R}^{N}$ and $\|\cdot\|$ denotes the usual Euclidean norm in $\mathbb{R}^{N}$. Moreover,

$$
\beta_{1}(x) \beta_{2}(a, y) \geq \beta(a, x, y) \geq \epsilon \beta_{3}(a, y)>0
$$

for all $a \in\left[0, a^{+}\right]$and $x, y \in \bar{\Omega}$, where $\beta_{1} \in Z_{+}$and $\beta_{2}, \beta_{3} \in L_{+}^{\infty}\left(\left(0, a^{+}\right) \times \Omega\right)$ and $\epsilon \in(0,1)$.

Let $P^{*}(a, x)$ be the steady state solution of system (3.1). Then it satisfies

$$
\begin{cases}\partial_{a} P^{*}(a, x)=d\left[\mathcal{L} P^{*}\right](a, x)-\mu(a, x) P^{*}(a, x), & a \in\left(0, a^{+}\right], x \in \bar{\Omega} \\ P^{*}(0, x)=\int_{0}^{a^{+}} \int_{\Omega} \beta(a, x, y) P^{*}(a, y) d y d a, & x \in \bar{\Omega} .\end{cases}
$$

By Lemma 2.1, we obtain $P^{*}(a, x)=\left(\mathcal{F}(0, a) P^{*}(0)\right)(x)$, where $\mathcal{F}$ is the solution operator of (2.1) with $f$ being replaced by $\mu$. Substituting it into the second equation of (3.2), one obtains the following integral equation:

$$
P^{*}(0, x)=\int_{0}^{a^{+}} \int_{\Omega} \beta(a, x, y)\left(\mathcal{F}(0, a) P^{*}(0)\right)(y) d y d a .
$$

In order to investigate the existence of a nontrivial solution of (3.3), we define a linear operator $K$ on $L^{1}(\Omega)$ by

$$
[K \psi](x):=\int_{0}^{a^{+}} \int_{\Omega} \beta(a, x, y)(\mathcal{F}(0, a) \psi)(y) d y d a, \quad \psi \in Z,
$$

and investigate the existence of a nontrivial fixed point $\psi^{*}$ of $K$. We have the following proposition. We mention that its proof is very similar to those of Lemma 4.1 and Proposition 4.2 in the later sections, thus here we only provide a sketch proof. 
Proposition 3.2 Let Assumption 3.1 be satisfied and $K$ be defined by (3.4). If $r(K)=1$, then $K$ has a positive fixed point $\psi^{*} \in Z_{+} \backslash\{0\}:=L_{+}^{1}(\Omega) \backslash\{0\}$. Here, $r(\cdot)$ denotes the spectral radius of an operator.

Proof Under Assumption 3.1, we can see from the well-known compactness criterion in $L^{1}$ (see Brezis (2010, Theorem 4.26)) that $K$ is a compact operator (see (Kang and Ruan 2021b, Lemma 3.3)). Moreover, $K$ is nonsupporting due to the positive lower bound of $\beta$, see Assumption 3.1-(ii). Then using the Krein-Rutman theorem (see Sawashima (1964) or Marek (1970)), we see that $r(K)$ is an eigenvalue of $K$ and there exists a corresponding positive nonzero eigenvector $\psi^{*} \in L_{+}^{1}(\Omega) \backslash\{0\}$. Thus we have $K \psi^{*}=r(K) \psi^{*}$. Since $r(K)=1$, the proof is complete.

The spectral radius $r(K)$ is thought to be the demographic reproduction number and the condition $r(K)=1$ corresponds to the situation where the growth rate is 0 in the well-known Lotka's characteristic equation. In fact, if the birth rate is separable such that $\beta(a, x, y)=\beta_{1}(x) \beta_{2}(a, y)$ with $\beta_{1} \in L_{+}^{1}(\Omega) \backslash\{0\}$ and $\beta_{2} \in L_{+}^{\infty}\left(\left(0, a^{+}\right) \times \Omega\right)$, then (3.4) can be rewritten as

$$
[K \psi](x)=\beta_{1}(x) \int_{0}^{a^{+}} \int_{\Omega} \beta_{2}(a, y)(\mathcal{F}(0, a) \psi)(y) d y d a, \quad \psi \in Z,
$$

and the range of $K$ becomes one-dimensional. Therefore, $\psi(x)=\beta_{1}(x)$ is a positive nonzero eigenvector of $K$ such that

$$
\left[K \beta_{1}\right](x)=\int_{0}^{a^{+}} \int_{\Omega} \beta_{2}(a, y)\left(\mathcal{F}(0, a) \beta_{1}\right)(y) d y d a \beta_{1}(x)=r(K) \beta_{1}(x),
$$

and hence, $r(K)=1$ implies that the following Lotka's characteristic equation holds:

$$
\int_{0}^{a^{+}} \int_{\Omega} \beta_{2}(a, y)\left(\mathcal{F}(0, a) \beta_{1}\right)(y) d y d a=1 .
$$

Once the existence of a positive fixed point $\psi^{*}(x)$ of operator $K$ is guaranteed from Proposition 3.2, it is not other than the nontrivial solution $P^{*}(0, x)$ of integral equation (3.3) and thus, the existence of a nontrivial steady state solution $P^{*} \in X_{+} \backslash\{0\}$ of system (3.1) is shown. Therefore, in the rest of this paper, we assume that $r(K)=1$ and hence system (3.1) has a nontrivial steady state solution $P^{*} \in X_{+} \backslash\{0\}$. Further, we assume that such a steady state has been attained from the initial time and bounded; that is,

$$
P(t, a, x) \equiv P^{*}(a, x), t \geq 0, \text { and } \sup _{(a, x) \in\left(0, a^{+}\right) \times \Omega} P^{*}(a, x)<\infty .
$$

\subsection{SIS epidemic model}

Now we consider model (1.1). Since it follows from $P(t, a, x)=P^{*}(a, x)$ for all $t \geq 0$ that $S(t, a, x)=P^{*}(a, x)-I(t, a, x)$, system (1.1) can be reduced to a single 
equation for $I(t, a, x)$ as

$$
\begin{cases}\left(\partial_{t}+\partial_{a}\right) I(t, a, x)=d[\mathcal{L} I](t, a, x)+\lambda(t, a, x)\left(P^{*}(a, x)-I(t, a, x)\right)- & (\gamma(a, x)+\mu(a, x)) I(t, a, x), \\ I(t, 0, x)=0, & t>0, a \in\left(0, a^{+}\right], x \in \bar{\Omega}, \\ I(0, a, x)=I_{0}(a, x) \in X_{+}, & t>0, x \in \bar{\Omega} \\ & a \in\left[0, a^{+}\right], x \in \bar{\Omega},\end{cases}
$$

where $\lambda(t, a, x)=\int_{0}^{a^{+}} \int_{\Omega} k(a, \sigma, x, y) I(t, \sigma, y) d y d \sigma$.

Now let us define a linear operator $A: D(A) \subset X \rightarrow X$ as

$$
\begin{aligned}
& A \psi:=-\partial_{a} \psi+d \mathcal{L} \psi-\mu \psi \\
& D(A)=\left\{\psi \in X: \psi \in W^{1,1}\left(\left(0, a^{+}\right), L^{1}(\Omega)\right), \psi(0, x)=0\right\} .
\end{aligned}
$$

Note that $\psi(0, x)$ is well-defined for any $\psi \in W^{1,1}\left(\left(0, a^{+}\right), L^{1}(\Omega)\right)$ since the Sobolev embedding $W^{1,1}\left(\left(0, a^{+}\right), L^{1}(\Omega)\right) \subset C\left(\left[0, a^{+}\right], L^{1}(\Omega)\right)$ holds. Let $E$ be a closed convex set defined by

$$
E:=\left\{\psi \in X_{+}: 0 \leq \psi(a, x) \leq P^{*}(a, x), \text { a.e. }\right\}
$$

Define $F: E \subset X \rightarrow X$ of nonlinear bounded operators by

$$
F(\psi)(a, x):=\lambda(a, x \mid \psi)\left(P^{*}(a, x)-\psi(a, x)\right)-\gamma(a, x) \psi(a, x), \quad \psi \in E,
$$

where

$$
\lambda(a, x \mid \psi):=\int_{0}^{a^{+}} \int_{\Omega} k(a, \sigma, x, y) \psi(\sigma, y) d y d \sigma .
$$

Note that $\lambda(\cdot, \cdot \mid \psi) \in L_{+}^{\infty}\left(\left(0, a^{+}\right) \times \Omega\right)$ for a fixed $\psi \in E$ by Assumption 2.2-(iv), (v). Then system (3.5) can be rewritten as a semilinear Cauchy problem

$$
\frac{d}{d t} I(t)=A I(t)+F(I(t)), \quad I(0)=I_{0} .
$$

We can see that operator $A$ is the infinitesimal generator of a $C_{0}$-semigroup $\left\{e^{t A}\right\}_{t \geq 0}$ defined by

$$
\left(e^{t A} \psi\right)(a, x)= \begin{cases}(\mathcal{F}(a-t, a) \psi(a-t))(x), & t<a \\ 0, & t \geq a\end{cases}
$$

where $\{\mathcal{F}(\sigma, a)\}_{0 \leq \sigma \leq a \leq a^{+}}$is defined in Lemma 2.1 via replacing $f$ by $\mu$. From (3.10) we immediately have $e^{t A} E \subset E$ and if $\psi \leq \phi$, then $e^{t A} \psi \leq e^{t A} \phi$ since the comparison principle of operators $\mathcal{F}(\sigma, a)$ for any $0 \leq \sigma \leq a \leq a^{+}$holds, where $\leq$denotes the usual ordering in $L^{1}\left(\left(0, a^{+}\right), Z\right)$. 
We also note that if $\alpha>0$ the resolvent $(I-\alpha A)^{-1}$ is given by the formula:

$$
\left[(I-\alpha A)^{-1} f\right](a, x)=\frac{1}{\alpha} \int_{0}^{a} e^{-\frac{1}{\alpha}(a-s)}(\mathcal{F}(s, a) f(s))(x) d s .
$$

Then again by the comparison theorem for $\{\mathcal{F}(\sigma, a)\}_{0 \leq \sigma \leq a \leq a^{+}}$, we have that

$$
\begin{aligned}
& (I-\alpha A)^{-1} E \subset E, \\
& \psi \leq \phi \Rightarrow(I-\alpha A)^{-1} \psi \leq(I-\alpha A)^{-1} \phi, \quad \forall \psi, \phi \in E .
\end{aligned}
$$

In fact, we can check that $u \equiv P^{*}(a, x)$ and $u \equiv 0$ are a pair of super-solution and sub-solution of the following nonlocal problem with Dirichlet boundary condition

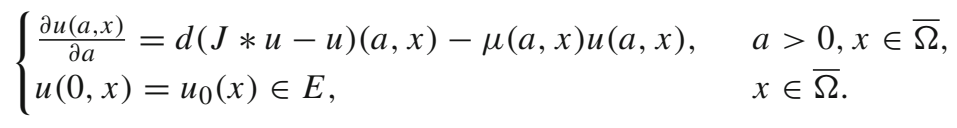

Thus $\mathcal{F}(s, a) f(s, x) \in E$ for any $f \in E$. Using the fact that $\gamma$ and $\lambda$ are a.e. bounded and the same kind of argument as in Busenberg et al. (1991, Proposition 3.1), it is easy to show the following proposition.

Proposition 3.3 Let Assumption 2.2 be satisfied. Then the nonlinear bounded operator $F: E \rightarrow X$ defined in (3.8) is Lipschitz continuous and there exists a constant $\alpha \in(0,1)$ such that if $\psi \in E$ then $\psi+\alpha F(\psi) \in E$, and if $\psi \leq \phi$ then $\psi+\alpha F(\psi) \leq$ $\phi+\alpha F(\phi)$.

Note that in the proof of Proposition 3.3, we will obtain $\alpha<\frac{1}{\gamma^{+}+\lambda^{+}}$, where $\lambda^{+}$is the upper bound of $\lambda$; that is, $\lambda[a, x \mid \psi]<\lambda^{+}$for any $\psi \in E$ in $\left[0, a^{+}\right] \times \bar{\Omega}$. Note that $\lambda^{+}$can be obtained by

$$
\lambda^{+}:=k^{+} \int_{0}^{a^{+}} \int_{\Omega} k_{2}(\sigma, y) P^{*}(\sigma, y) d y d \sigma .
$$

Using $\alpha$ appeared in Proposition 3.3, we can write problem (3.9) as

$$
\frac{d}{d t} I(t)=\left(A-\frac{1}{\alpha}\right) I(t)+\frac{1}{\alpha}\left(I(t)+\alpha F(I(t)), \quad I(0)=I_{0} .\right.
$$

Now we are in a position to look for a mild solution $I \in E$ of (3.14), which is given by a solution of the integral equation

$$
I(t)=e^{-\frac{1}{\alpha} t} e^{t A} I_{0}+\frac{1}{\alpha} \int_{0}^{t} e^{-\frac{1}{\alpha}(t-s)} e^{(t-s) A}[I(s)+\alpha F(I(s))] d s .
$$

Equation (3.15) provides the following scheme of the standard iterative procedure for obtaining a mild solution $I \in E$ :

$$
I^{0}(t)=I_{0},
$$




$$
\begin{aligned}
& I^{n+1}(t)=e^{-\frac{1}{\alpha} t} e^{t A} I_{0}+\frac{1}{\alpha} \int_{0}^{t} e^{-\frac{1}{\alpha}(t-s)} e^{(t-s) A}\left[I^{n}(s)+\alpha F\left(I^{n}(s)\right)\right] d s, \\
& \quad n=0,1,2, \ldots
\end{aligned}
$$

Since we can see that $E$ is invariant with respect to the iteration process, that is $I^{n+1} \in$ $E$ if $I^{n} \in E$, according to the argument in Busenberg et al. (1991, Theorem 3.2), we can prove the following theorem.

Theorem 3.4 Let Assumption 2.2 be satisfied and $I_{0} \in$ E. Then Eq. (3.9) has a unique mild solution $I(t)=\mathcal{S}(t) I_{0} \in C([0, \infty), E)$, where $\{\mathcal{S}(t)\}_{t \geq 0}$ defines a $C_{0}$-semigroup in $X$ with the following properties:

(i) $\mathcal{S}(t) E \subset E$;

(ii) If $I_{0} \leq I_{1}$, then $\mathcal{S}(t) I_{0} \leq \mathcal{S}(t) I_{1}$;

(iii) If $\xi \in(0,1)$, then $\xi \mathcal{S}(t) I_{0} \leq \mathcal{S}(t)\left(\xi I_{0}\right)$.

Proof For the completeness, we provide the proof based on Busenberg et al. (1991, Theorem 3.2). In fact, (i) and (ii) can be obtained easily by the arguments before the theorem. Thus we are only concerned on (iii). Let $I_{\xi}^{n}$ be the iterates (3.16) with $I_{0}$ replaced by $\xi I_{0}$. Since $0 \leq \xi \leq 1$, we have

$$
\xi I+\alpha F(\xi I) \geq \xi(I+\alpha F(I)),
$$

so that

$$
I_{\xi}^{n} \geq \xi I^{n}
$$

and going to the limit, we obtain (iii).

\section{Existence of nontrivial steady states}

Now in this section we investigate the existence of steady states of (3.5). Let $I^{*}(a, x)$ be a (time-independent) steady states solution of system (3.5). Then it satisfies

$$
\begin{cases}\partial_{a} I^{*}(a, x)=d\left[\mathcal{L} I^{*}\right](a, x)+\lambda^{*}(a, x)\left(P^{*}(a, x)-I^{*}(a, x)\right)-(\gamma+\mu) I^{*}(a, x), & a \in\left(0, a^{+}\right], x \in \bar{\Omega}, \\ I^{*}(0, x)=0, & x \in \bar{\Omega}, \\ \lambda^{*}(a, x)=\int_{0}^{a^{+}} \int_{\Omega} k(a, \sigma, x, y) I^{*}(\sigma, y) d y d \sigma, & a \in\left(0, a^{+}\right], x \in \bar{\Omega} .\end{cases}
$$

It is obvious that the trivial disease-free steady state solution $I^{*}(a, x) \equiv 0$ always exists. In what follows, we investigate the existence of a nontrivial endemic steady state solution $I^{*} \in E \backslash\{0\}$, where $E$ is defined in (3.7).

Considering the linearized equation of (4.1) at $I^{*}=0$, we have

$$
\begin{cases}\partial_{a} I^{*}(a, x)=d\left[\mathcal{L} I^{*}\right](a, x)+\lambda^{*}(a, x) P^{*}(a, x)-(\gamma+\mu) I^{*}(a, x), & a \in\left(0, a^{+}\right], x \in \bar{\Omega} \\ I^{*}(0, x)=0, & x \in \bar{\Omega}, \\ \lambda^{*}(a, x)=\int_{0}^{a^{+}} \int_{\Omega} k(a, \sigma, x, y) I^{*}(\sigma, y) d y d \sigma, & a \in\left(0, a^{+}\right], x \in \bar{\Omega}\end{cases}
$$


Viewing $f(a, x)=\lambda^{*}(a, x)+\gamma(a, x)+\mu(a, x)$ in Lemma 2.1 and solving the first equation of (4.1), we obtain

$$
I^{*}(a, x)=\int_{0}^{a}\left(\mathcal{F}_{\lambda^{*}}(\sigma, a)\left(\lambda^{*} P^{*}\right)(\sigma)\right)(x) d \sigma
$$

where $\mathcal{F}_{\lambda^{*}}$ is the solution operator of (4.1) as in Lemma 2.1 with $f$ being replaced by $\lambda^{*}+\gamma+\mu$ (note that $\lambda^{*}+\gamma+\mu \in L_{+}^{\infty}\left(\left(0, a^{+}\right) \times \Omega\right)$ ). Substituting (4.3) into the third equation of (4.1), we obtain the following integral equation of $\lambda^{*}(a, x)$ :

$$
\lambda^{*}(a, x)=\int_{0}^{a^{+}} \int_{\Omega} k(a, \sigma, x, y) \int_{0}^{\sigma}\left(\mathcal{F}_{\lambda^{*}}(\tau, \sigma)\left(\lambda^{*} P^{*}\right)(\tau)\right)(y) d \tau d y d \sigma .
$$

Solving the first equation of (4.2), we obtain

$$
I^{*}(a, x)=\int_{0}^{a}\left(\mathcal{F}_{0}(\sigma, a)\left(\lambda^{*} P^{*}\right)(\sigma)\right)(x) d \sigma,
$$

where $\mathcal{F}_{0}$ is the solution operator of (4.1) as in Lemma 2.1 with $f$ being replaced by $\gamma+\mu$. Substituting (4.5) into the third equation of (4.2), we obtain the following integral equation of $\lambda^{*}(a, x)$ :

$$
\lambda^{*}(a, x)=\int_{0}^{a^{+}} \int_{\Omega} k(a, \sigma, x, y) \int_{0}^{\sigma}\left(\mathcal{F}_{0}(\tau, \sigma)\left(\lambda^{*} P^{*}\right)(\tau)\right)(y) d \tau d y d \sigma .
$$

Observe that we have $\mathcal{F}_{\lambda^{*}}(\sigma, a) \leq \mathcal{F}_{0}(\sigma, a)$ for all $0 \leq \sigma \leq a \leq a^{+}$in the operator sense by Lemma 2.1. Now we define a nonlinear operator $\Phi$ on $X$ by

$$
[\Phi \phi](a, x):=\int_{0}^{a^{+}} \int_{\Omega} k(a, \sigma, x, y) \int_{0}^{\sigma}\left(\mathcal{F}_{\phi}(\tau, \sigma)\left(\phi P^{*}\right)(\tau)\right)(y) d \tau d y d \sigma, \quad \phi \in X,
$$

and investigate the existence of a nontrivial positive fixed point $\phi^{*} \in X_{+} \backslash\{0\}$ such that $\Phi\left(\phi^{*}\right)=\phi^{*}$, which is no other than the desired nontrivial positive solution $\lambda^{*} \in X_{+} \backslash\{0\}$ of (4.4).

We can check that $\Phi$ is bounded and positive by Assumption 2.2 and the boundedness of $P^{*}$. Next, consider the following linear integral operator $M$ on $X$ :

$$
[M \phi](a, x):=\int_{0}^{a^{+}} \int_{\Omega} k(a, \sigma, x, y) \int_{0}^{\sigma}\left(\mathcal{F}_{0}(\tau, \sigma)\left(\phi P^{*}\right)(\tau)\right)(y) d \tau d y d \sigma, \quad \phi \in X
$$

One can see that $M$ is the Fréchert derivative of $\Phi$ at $\phi=0$ and a positive linear majorant of $\Phi$. We will show that the spectral radius $r(M)$ of operator $M$ plays the role of a threshold value for the existence of a nontrivial fixed point of operator $\Phi$; that is, if $r(M)>1$, then $\Phi$ has a positive nontrivial fixed point. We prove the following lemma about $M$. 
Lemma 4.1 Let Assumption 2.2 be satisfied and $M$ be defined by (4.8). Then $M$ is positive, bounded, compact and nonsupporting.

Proof First we can see that $M$ is positive since $\left\{\mathcal{F}_{0}(\tau, \sigma)\right\}_{0 \leq \tau \leq a}$ is positive. Next we prove the compactness. Note by Assumption 2.2-(i) that for $\phi \in \mathcal{B}$, where $\mathcal{B}$ is a uniformly bounded subset in $X$,

$$
\begin{aligned}
\| & {[M \phi](\cdot+\kappa, \cdot+h)-[M \phi](\cdot, \cdot) \|_{X} } \\
\leq & \int_{0}^{a^{+}} \int_{\Omega}\left[\int_{0}^{a^{+}} \int_{\Omega}|k(a+\kappa, \sigma, x+h, y)-k(a, \sigma, x, y)|\right. \\
& \left.\times \int_{0}^{\sigma}\left(\mathcal{F}_{0}(\sigma, \tau)\left(\phi P^{*}\right)(\tau)\right)(y) d \tau d y d \sigma\right] d x d a \\
\leq & \int_{0}^{a^{+}} \int_{\Omega}\left[\int_{0}^{a^{+}} \int_{\Omega}|k(a+\kappa, \sigma, x+h, y)-k(a, \sigma, x, y)| d x d a\right] \\
& \times \int_{0}^{\sigma}\left(\mathcal{F}_{0}(\sigma, \tau)\left(\phi P^{*}\right)(\tau)\right)(y) d \tau d y d \sigma \\
\rightarrow & 0 \text { as }\|(\kappa, h)\| \rightarrow 0
\end{aligned}
$$

where we used Minkowski inequality and Assumption 2.2-(iii). Thus $M: X \rightarrow X$ is compact by Kolmogorov compactness theorem, see Brezis (2010, Theorem 4.26). Finally, we prove that $M$ is nonsupporting. By Assumption 2.2-(iv), we can define the following linear functional $f_{0} \in X^{*}$ on $X$,

$$
\left\langle f_{0}, \phi\right\rangle:=\int_{0}^{a^{+}} \int_{\Omega} \epsilon k^{-} k_{2}(\sigma, y) \int_{0}^{\sigma}\left(\mathcal{F}_{0}(\tau, \sigma)\left(\phi P^{*}\right)(\tau)\right)(y) d \tau d y d \sigma
$$

and hence we see from the positivity of $P^{*}, k^{-}$and $\left\{\mathcal{F}_{0}(\tau, \sigma)\right\}_{0 \leq \tau \leq \sigma \leq a^{+}}$that the functional $f_{0}$ is strictly positive (that is, $\left\langle f_{0}, \phi\right\rangle>0$ for all $\phi \in X_{+} \backslash\{0\}$ ). Now we have

$$
M \phi \geq\left\langle f_{0}, \phi\right\rangle e, \quad e(a, x) \equiv 1, \quad \forall(a, x) \in\left[0, a^{+}\right] \times \Omega .
$$

Note that $e \in X_{+}$, then we have

$$
\begin{aligned}
M^{n+1} \phi & \geq\left\langle f_{0}, M^{n} \phi\right\rangle e \\
& \geq\left\langle f_{0},\left\langle f_{0}, M^{n-1} \phi\right\rangle e\right\rangle e \\
& =\left\langle f_{0}, e\right\rangle\left\langle f_{0}, M^{n-1} \phi\right\rangle e \\
& \geq\left\langle f_{0}, e\right\rangle^{n}\left\langle f_{0}, \phi\right\rangle e, \quad n=1,2, \ldots
\end{aligned}
$$

Thus, for any $M^{*} \in X_{+}^{*} \backslash\{0\}$ and $\phi \in X_{+} \backslash\{0\}$, it follows that

$$
\left\langle M^{*}, M^{n} \phi\right\rangle \geq\left\langle f_{0}, e\right\rangle^{n-1}\left\langle f_{0}, \phi\right\rangle\left\langle M^{*}, e\right\rangle>0, \quad n=1,2, \ldots,
$$


which implies that $M$ is nonsupporting. The proof is complete.

Similar to Lemma 4.1, one can show that $\Phi$ defined by (4.7) is also compact. Under Lemma 4.1, we can apply the fixed point theorem (Theorem 2.3) to prove the following proposition.

Proposition 4.2 Let Assumption 2.2 be satisfied and $\Phi, M$ be defined by (4.7) and (4.8), respectively.

(i) If $r(M) \leq 1$, then $\Phi$ only has the trivial fixed point $\phi \equiv 0$ in $X_{+}$;

(ii) If $r(M)>1$, then $\Phi$ has at least one nontrivial fixed point $\phi^{*} \in X_{+} \backslash\{0\}$.

Proof First note that conditions (i) and (ii) of Theorem 2.3 are obviously satisfied. From Lemma 4.1, we can apply the theory of nonsupporting operators (see Inaba (1990) or Marek (1970)) to prove that $r(M)>1$ is an eigenvalue of operator $M$ and there exists a corresponding positive nonzero eigenvector and $M$ does not have any eigenvector associated with eigenvalue 1 . Hence, conditions (iii) and (iv) of Theorem 2.3 follow and, consequently, $\Phi$ has at least one nontrivial fixed point in $X_{+}$.

Next, we prove (i). From Lemma 4.1, we see that $M$ is compact and nonsupporting. Since $\Phi$ is a positive operator from the positive cone $X_{+}$to itself and $M$ is the positive linear majorant of $\Phi$, we can apply Theorem 2.4 to conclude that $\Phi$ has no nontrivial fixed point in $X_{+}$provided $r(M) \leq 1$. This completes the proof.

The existence of a nontrivial fixed point of $\Phi$ implies the existence of a nontrivial steady state solution $I^{*} \in X_{+} \backslash\{0\}$; that is, the existence of a nontrivial steady state solution $\left(S^{*}, I^{*}\right) \in X_{+}^{2} \backslash\{0,0\}$ of the original system (1.1). Further, $I^{*}$ must be in $E \backslash\{0\}$, otherwise $I^{*} \equiv 0$ from (4.1). In conclusion, from Proposition 4.2, the following theorem can be obtained as one of the main results of this paper.

Theorem 4.3 Let Assumption 2.2 be satisfied and $M$ be defined in (4.8).

(i) If $r(M) \leq 1$, then system $(1.1)$ only has the trivial steady state $\left(S^{*}, I^{*}\right)=\left(P^{*}, 0\right)$ in $X_{+}^{2}$;

(ii) If $r(M)>1$, then system (1.1) has at least one nontrivial steady state $\left(S^{*}, I^{*}\right)$ in $X_{+}^{2}$.

\section{Basic reproduction number}

In this section we show that the threshold value $r(M)$ obtained in the previous section is equal to the basic reproduction number $\mathcal{R}_{0}$ for system (1.1). Following the idea from Diekmann et al. (1990) and Kuniya and Oizumi (2015), linearizing system (3.5) at the disease-free steady state, we obtain the following system

$$
\begin{cases}\left(\partial_{t}+\partial_{a}\right) I(t, a, x)=d[\mathcal{L} I](t, a, x)+v(t, a, x)-(\gamma+\mu) I(t, a, x), & t>0, a \in\left(0, a^{+}\right], x \in \bar{\Omega}, \\ I(t, 0, x)=0, & t>0, x \in \bar{\Omega} \\ I(0, a, x)=I_{0}(a, x), & a \in\left[0, a^{+}\right], x \in \bar{\Omega},\end{cases}
$$


where $v(t, a, x)=P^{*}(a, x) \int_{0}^{a^{+}} \int_{\Omega} k(a, \sigma, x, y) I(t, \sigma, y) d y d \sigma$ denotes the density of newly infected population at the linear invasion phase. Integrating along the characteristic line $t-a=$ constant, we obtain

$$
I(t, a, x)=\int_{0}^{a}\left(\mathcal{F}_{0}(\sigma, a) v(t-a+\sigma, \sigma)\right)(x) d \sigma .
$$

Here note that it is suffices to consider $t>a^{+}$from Assumption 2.2-(ii). Now plugging it into the equation of $v$, we have

$$
\begin{aligned}
v(t, a, x) & =P^{*}(a, x) \int_{0}^{a^{+}} \int_{\Omega} k(a, \sigma, x, y) \int_{0}^{\sigma}\left(\mathcal{F}_{0}(\tau, \sigma) v(t-\sigma+\tau, \tau)\right)(y) d \tau d y d \sigma \\
& =P^{*}(a, x) \int_{0}^{a^{+}} \int_{\Omega} k(a, \sigma, x, y) \int_{0}^{\sigma}\left(\mathcal{F}_{0}(\sigma-\rho, \sigma) v(t-\rho, \sigma-\rho)\right)(y) d \rho d y d \sigma \\
& =P^{*}(a, x) \int_{\Omega} \int_{0}^{a^{+}} \int_{\rho}^{a^{+}} k(a, \sigma, x, y)\left(\mathcal{F}_{0}(\sigma-\rho, \sigma) v(t-\rho, \sigma-\rho)\right)(y) d \sigma d \rho d y .
\end{aligned}
$$

Defining a linear operator $B(\rho, x, y): X \rightarrow L^{1}\left(0, a^{+}\right)$by

$[B(\rho, x, y) \varphi(\cdot, y)](a):=\int_{\rho}^{a^{+}} k(a, \sigma, x, y)\left(\mathcal{F}_{0}(\sigma-\rho, \sigma) \varphi(\sigma-\rho)\right)(y) d \sigma, \quad \varphi \in X$,

we can rewrite (5.2) as the abstract integral equation in $X$ :

$$
v(t, x)=P^{*}(\cdot, x) \int_{\Omega} \int_{0}^{a^{+}} B(\rho, x, y) v(t-\rho, y) d \rho d y .
$$

Then following the arguments in Diekmann et al. (1990), we obtain the next generation operator in $X$ as

$$
\begin{aligned}
{[\mathcal{G} \varphi](a, x):=} & P^{*}(a, x) \int_{\Omega} \int_{0}^{a^{+}}[B(\rho, x, y) \varphi](a) d \rho d y \\
= & P^{*}(a, x) \int_{\Omega} \int_{0}^{a^{+}} \int_{\rho}^{a^{+}} k(a, \sigma, x, y) \\
& \times\left(\mathcal{F}_{0}(\sigma-\rho, \sigma) \varphi(\sigma-\rho)\right)(y) d \sigma d \rho d y
\end{aligned}
$$

and the basic reproduction number $\mathcal{R}_{0}$ is defined by its spectral radius

$$
\mathcal{R}_{0}:=r(\mathcal{G})
$$

Then we have the following proposition inspired from Kuniya and Oizumi (2015, Proposition 4.1). 
Proposition 5.1 Let $M, \mathcal{G}$ and $\mathcal{R}_{0}$ be defined in (4.8), (5.3) and (5.4) respectively. It follows that

$$
r(M)=r(\mathcal{G})=\mathcal{R}_{0}
$$

Proof Considering the calculation of (5.2) conversely, we see that the right hand side of (5.2) is

$$
[\mathcal{G} \varphi](a, x)=P^{*}(a, x) \int_{0}^{a^{+}} \int_{\Omega} k(a, \sigma, x, y) \int_{0}^{\sigma}\left(\mathcal{F}_{0}(\tau, \sigma) \varphi(\tau)\right)(y) d \tau d y d \sigma
$$

Let us define a linear operator $Q$ on $X$ by

$$
[Q \psi](a, x)=\psi(a, x) P^{*}(a, x), \quad \psi \in X
$$

Then, comparing (4.8) with (5.5), we see that

$$
M=Q^{-1} \mathcal{G} Q
$$

Since $r\left(Q^{-1} \mathcal{G} Q\right)=r(\mathcal{G})=\mathcal{R}_{0}$, we obtain $r(M)=r(\mathcal{G})=\mathcal{R}_{0}$.

Theorem 5.2 Let Assumption 2.2 be satisfied and $\mathcal{R}_{0}$ be the basic reproduction number defined in (5.4).

(i) If $\mathcal{R}_{0} \leq 1$, then system (1.1) has only the trivial steady state $\left(S^{*}, I^{*}\right)=\left(P^{*}, 0\right)$ in $X_{+}^{2}$;

(ii) If $\mathcal{R}_{0}>1$, then system (1.1) has at least one nontrivial steady state $\left(S^{*}, I^{*}\right)$ in $X_{+}^{2}$.

\section{Uniqueness of the nontrivial steady state}

In this section we discuss the uniqueness of the nontrivial steady state $I^{*} \in E$. Note that $I^{*}$ is a steady state of (3.5) if and only if it satisfies

$$
T I^{*}:=(I-\alpha A)^{-1}(I+\alpha F) I^{*}=I^{*} .
$$

From (3.12) and Proposition 3.3, we can see that $T(E) \subset E$ and $T \psi \leq T \phi$ for any $\psi \leq \phi$ and $\psi, \phi \in E$.

We need several lemmas to prove the uniqueness. First we state a necessary condition which should be satisfied by any nontrivial solution of (6.1).

Lemma 6.1 Let $I^{*}$ be a nontrivial solution of (6.1). Then

$$
\int_{0}^{a^{+}} \int_{\Omega} k_{2}(\sigma, y) I^{*}(\sigma, y) d y d \sigma>0
$$


Proof If (6.2) is not satisfied, then

$$
\int_{0}^{a^{+}} \int_{\Omega} k(a, \sigma, x, y) I^{*}(\sigma, y) d y d \sigma=0, \quad \forall(a, x) \in\left(0, a^{+}\right) \times \bar{\Omega},
$$

and $I^{*}$ satisfies the following equations

$$
\begin{cases}\partial_{a} I^{*}(a, x)=d \mathcal{L} I^{*}(a, x)-(\gamma(a, x)+\mu(a, x)) I^{*}(a, x), & a \in\left(0, a^{+}\right], x \in \bar{\Omega} \\ I^{*}(0, x)=0, & x \in \bar{\Omega} .\end{cases}
$$

Thus $I^{*}$ is identically zero by the uniqueness of solution for the above linear equation, which is a contradiction.

We next give an estimate on the nontrivial fixed point of $T$.

Lemma 6.2 Let Assumption 2.2 be satisfied and $I^{*}$ be a nontrivial solution to (6.1). Then

$$
\epsilon_{1} m(a, x) \leq I^{*}(a, x) \leq \epsilon_{2} m(a, x)
$$

where

$$
m(a, x):=\int_{0}^{a}\left(\mathcal{F}(\sigma, a)\left(k_{1} P^{*}\right)(\sigma)\right)(x) d \sigma,
$$

$\epsilon_{1}$ and $\epsilon_{2}$ are positive constants depending on $I^{*}$ and $\{\mathcal{F}(\sigma, a)\}_{0 \leq \sigma \leq a \leq a^{+}}$is from Lemma 2.1 via replacing $f$ by $\mu$.

Proof As $I^{*}$ is a nontrivial solution, (6.2) is satisfied so that

$$
c_{1} k_{1}(a, x) \leq \lambda\left[a, x \mid I^{*}\right] \leq c_{2} k_{1}(a, x),
$$

where $c_{1}, c_{2}$ are positive. Hence $I^{*}$ satisfies the inequality

$$
\begin{aligned}
& c_{1} k_{1}(a, x) P^{*}(a, x)-\left(\lambda^{+}+\gamma^{+}\right) I^{*}(a, x) \\
& \quad \leq \partial_{a} I^{*}(a, x)-d \mathcal{L} I^{*}(a, x)+\mu(a, x) I^{*}(a, x) \\
& \quad \leq c_{2} k_{1}(a, x) P^{*}(a, x) .
\end{aligned}
$$

Since $I^{*}(0, x)=0$, we can find positive $\epsilon_{1}, \epsilon_{2}$ such that (6.4) is satisfied.

Lemma 6.3 Let Assumption 2.2 be satisfied and $I^{*} \in E$ be a nontrivial fixed point of $T$. Let $\xi \in(0,1)$ and $I \in E$ such that $\xi I^{*}+I \in E$ and $\int_{0}^{a^{+}} \int_{\Omega} k_{2}(\sigma, y) I(\sigma, y) d y d \sigma>0$. Then there exists a constant $\epsilon_{3}>0$ such that

$$
T\left(\xi I^{*}+I\right) \geq \xi I^{*}+\epsilon_{3}(1-\xi) m .
$$


Proof Observe that $\left(\alpha<\frac{1}{\gamma^{+}+\lambda^{+}}\right)$

$$
\begin{aligned}
\xi I^{*}+I+\alpha F\left(\xi I^{*}+I\right) \\
=\xi I^{*}+I+\alpha\left(P^{*}-\xi I^{*}-I\right) \lambda\left[\cdot, \cdot \mid \xi I^{*}+I\right]-\alpha \gamma\left(\xi I^{*}+I\right) \\
=\xi I^{*}+\alpha F\left(\xi I^{*}\right)+I\left(1-\alpha \lambda\left[\cdot, \cdot \mid \xi I^{*}+I\right]-\alpha \gamma\right)+\alpha\left(P^{*}-\xi I^{*}\right) \lambda[\cdot, \cdot \mid I] \\
\geq \xi I^{*}+\alpha \xi F\left(I^{*}\right)+\alpha\left(P^{*}-\xi I^{*}\right) \lambda[\cdot, \cdot \mid I] \\
\geq \xi\left(I^{*}+\alpha F\left(I^{*}\right)\right)+\alpha \epsilon\left(P^{*}-\xi I^{*}\right) k_{1} \int_{0}^{a^{+}} \int_{\Omega} k_{2}(\sigma, y) I(\sigma, y) d y d \sigma .
\end{aligned}
$$

Then from (3.11), (3.12) and (6.5), we obtain (6.7).

Lemma 6.4 Let Assumption 2.2 be satisfied and $I^{*} \in E$ be a nontrivial fixed point of $T$ and let $\xi \in(0,1)$. Then there exists a constant $\epsilon_{4}>0$ such that

$$
T\left(\xi I^{*}\right) \geq \xi I^{*}+\xi(1-\xi) \epsilon_{4} m_{1}
$$

where $m_{1}(a, x):=\int_{0}^{a}\left(\mathcal{F}(\sigma, a) I^{*}(\sigma)\right)(x) d \sigma$.

Proof Observe that

$$
\begin{aligned}
& \xi I^{*}+\alpha F\left(\xi I^{*}\right) \\
& \quad=\xi\left(I^{*}+\alpha F\left(I^{*}\right)\right)+\alpha \xi(1-\xi) I^{*} \lambda\left[\cdot, \cdot \mid I^{*}\right] \\
& \quad \geq \xi\left(I^{*}+\alpha F\left(I^{*}\right)\right)+\alpha \xi(1-\xi) \epsilon I^{*} k_{1} \int_{0}^{a^{+}} \int_{\Omega} k_{2}(\sigma, y) I^{*}(\sigma, y) d y d \sigma \\
& \quad \geq \xi\left(I^{*}+\alpha F\left(I^{*}\right)\right)+\alpha \xi(1-\xi) \epsilon I^{*} k^{-} \int_{0}^{a^{+}} \int_{\Omega} k_{2}(\sigma, y) I^{*}(\sigma, y) d y d \sigma .
\end{aligned}
$$

Then we have the desired (6.8) from (3.11), (3.12) and definition of $m_{1}$.

Theorem 6.5 Let Assumption 2.2 be satisfied, then $T$ has at most one nontrivial fixed point.

Proof Let $u^{*} \neq v^{*}$ be two nontrivial fixed points. Let $\xi=\sup \left\{k \in \mathbb{R}_{+}: v^{*} \geq k u^{*}\right\}$. From (6.4), we can see that $\xi>0$. If $\xi<1$, we have from Lemma 6.4 that

$$
v^{*}=T^{2}\left(v^{*}\right) \geq T^{2}\left(\xi u^{*}\right) \geq T\left(\xi u^{*}+\xi(1-\xi) \epsilon_{4} m_{1}\right) .
$$

If $\epsilon_{4}$ is small enough, we can assume that $\xi(1-\xi) \epsilon_{4} m_{1}, \xi u^{*}+\xi(1-\xi) \epsilon_{4} m_{1} \in E$. Then we can apply Lemma 6.3 (taking $I=\xi(1-\xi) \epsilon_{4} m_{1}$ ) to obtain

$$
v^{*} \geq T\left(\xi u^{*}+\xi(1-\xi) \epsilon_{4} m_{1}\right) \geq \xi u^{*}+(1-\xi) \epsilon_{3} m,
$$

which implies by (6.4) that

$$
v^{*} \geq \xi u^{*}+(1-\xi) \frac{\epsilon_{3}}{\epsilon_{2}} u^{*}
$$


But this contradicts the definition of $\xi$. Therefore, we have $\xi \geq 1$ and $v^{*} \geq u^{*}$. By using the same approach, we can show that $u^{*} \geq v^{*}$. Then $u^{*}=v^{*}$.

\section{Local stability of the nontrivial steady state}

\subsection{Local stability}

In this subsection we analyze local stability of the nontrivial steady state $I^{*} \in E$. Note that $I^{*} \in L_{+}^{\infty}\left(\left(0, a^{+}\right) \times \Omega\right)$ since $I^{*} \in E$ implies that $I^{*}(a, x) \leq P^{*}(a, x)$. Consider the linearization of system (4.1) at the nontrivial steady state $I^{*} \in E$ in the following

$$
\begin{cases}\left(\partial_{t}+\partial_{a}\right) I(t, a, x)=d[\mathcal{L} I](t, a, x)+\lambda(t, a, x \mid I)\left(P^{*}-I^{*}\right)(a, x)- & \lambda^{*}(a, x) I(t, a, x)-(\gamma+\mu) I(t, a, x), \\ & t>0, a \in\left(0, a^{+}\right], x \in \bar{\Omega}, \\ I(t, 0, x)=0, & t>0, x \in \bar{\Omega} \\ I(0, a, x)=I_{0}(a, x), & a \in\left[0, a^{+}\right], x \in \bar{\Omega},\end{cases}
$$

where $\lambda(t, a, x \mid I)=\int_{0}^{a^{+}} \int_{\Omega} k(a, \sigma, x, y) I(t, \sigma, y) d y d \sigma$. Define a linear operator $\mathcal{A}: D(\mathcal{A}) \subset X \rightarrow X$ as

$$
\begin{aligned}
& \mathcal{A} \psi:=-\partial_{a} \psi+d \mathcal{L} \psi-\lambda^{*}(a, x) \psi-(\gamma(a, x)+\mu(a, x)) \psi, \\
& D(\mathcal{A})=\left\{\psi \in X: \psi \in W^{1,1}\left(\left(0, a^{+}\right), L^{1}(\Omega)\right), \psi(0, x)=0\right\},
\end{aligned}
$$

where $\lambda^{*}(a, x)=\int_{0}^{a^{+}} \int_{\Omega} k(a, \sigma, x, y) I^{*}(\sigma, y) d y d \sigma$. Rewrite equation (7.1) as

$$
\frac{d}{d t} I(t)=\mathcal{A} I(t)+G(I(t)), \quad I(0)=I_{0},
$$

where $G: E \subset X \rightarrow E$ is defined as

$$
[G \psi](a, x):=\lambda(a, x \mid \psi)\left(P^{*}(a, x)-I^{*}(a, x)\right) .
$$

Then by using the same argument in Lemma 4.1, one can show that $G$ is compact. Now we can check that $\mathcal{A}$ generates a nilpotent semigroup and its perturbed semigroup by the compact operator $G$ is eventually compact. Hence, $\omega_{1}(\mathcal{A}+G)=\omega_{1}(\mathcal{A})=-\infty$, where $\omega_{1}(A)$ represents the essential growth bound of operator $A$. It follows that the stability of $I^{*}$ is determined by the eigenvalues of $\mathcal{A}+G$. By solving the characteristic equation $(\mathcal{A}+G) \psi=\Lambda \psi$ and viewing $f(a, x)=\lambda^{*}(a, x)+\gamma(a, x)+\mu(a, x)$ in Lemma 2.1, we obtain

$$
\psi(a, x)=\int_{0}^{a} e^{-\Lambda(a-\sigma)}\left(\mathcal{F}_{\lambda^{*}}(\sigma, a)\left(\lambda(\psi)\left(P^{*}-I^{*}\right)\right)(\sigma)\right)(x) d \sigma .
$$

Plugging (7.4) into the equation of $\lambda$, we have 


$$
\begin{aligned}
\lambda(a, x \mid \psi)= & \int_{0}^{a^{+}} \int_{\Omega} k(a, \sigma, x, y) \int_{0}^{\sigma} e^{-\Lambda(\sigma-\tau)} \\
& \times\left(\mathcal{F}_{\lambda^{*}}(\tau, \sigma)\left(\lambda(\psi)\left(P^{*}-I^{*}\right)\right)(\tau)\right)(y) d \tau d y d \sigma .
\end{aligned}
$$

Define an operator $\Gamma_{\Lambda}: X \rightarrow X$ by

$$
\Gamma_{\Lambda} \varphi:=\int_{0}^{a^{+}} \int_{\Omega} k(\cdot, \sigma, \cdot, y) \int_{0}^{\sigma} e^{-\Lambda(\sigma-\tau)}\left(\mathcal{F}_{\lambda^{*}}(\tau, \sigma)\left(\varphi\left(P^{*}-I^{*}\right)\right)(\tau)\right)(y) d \tau d y d \sigma .
$$

Here we should emphasize that $\mathcal{F}_{\lambda^{*}}(\tau, \sigma)$ is a linear operator since $\lambda^{*}$ is fixed now and that $\mathcal{F}_{\lambda^{*}}(\tau, \sigma)$ is applied to the whole $\varphi\left(P^{*}-I^{*}\right)(\tau, y)$ rather than only $\varphi(\tau, y)$. Note that $\Gamma_{\Lambda}$ is well defined due to the boundedness of $P^{*}$ and $I^{*}$. Then it is easy to check that $\Gamma_{\Lambda}$ is also compact and nonsupporting by Lemma 4.1, thus has a simple eigenvalue $r\left(\Gamma_{\Lambda}\right)$ corresponding to a simple and positive eigenvector. Moreover the mapping $\Lambda \rightarrow r\left(\Gamma_{\Lambda}\right): \mathbb{R} \rightarrow(0, \infty)$ is continuous and strictly decreasing from $+\infty$ to 0 (see Lemma 3.4 of Kang et al. (2020b)). This implies that there exists a unique $\Lambda_{0}$ associated with a positive eigenvector $\varphi_{0} \in X_{+}$such that $r\left(\Gamma_{\Lambda_{0}}\right)=1$. Thus $\Gamma_{\Lambda_{0}} \varphi_{0}=\varphi_{0}$ and hence $\Lambda_{0}$ is an eigenvalue of $\mathcal{A}+G$. In what follows, we will show that such $\Lambda_{0}$ is also a principal eigenvalue, i.e. $\Lambda_{0}>|\Lambda|$ for any other $\Lambda \in \sigma_{P}(\mathcal{A}+G) \backslash\left\{\Lambda_{0}\right\}$, where $\sigma_{P}(A)$ represents the point spectrum of $A$. Once it is true, we have the following theorem.

Theorem 7.1 Let Assumption 2.2 be satisfied. Then it is locally stable when $\Lambda_{0}<0$, which is equivalent to $r\left(\Gamma_{0}\right)<1$; Otherwise, $I^{*}$ is unstable when $\Lambda_{0}>0$, which is equivalent to $r\left(\Gamma_{0}\right)>1$.

Proof Since $\Gamma_{\Lambda}$ is compact, it follows that $\sigma\left(\Gamma_{\Lambda}\right) \backslash\{0\}=\sigma_{P}\left(\Gamma_{\Lambda}\right) \backslash\{0\}$. Note that $\left\{\Lambda \in \mathbb{C}: 1 \in \sigma_{P}\left(\Gamma_{\Lambda}\right)\right\}=\left\{\Lambda \in \mathbb{C}: \Lambda \in \sigma_{P}(\mathcal{A}+G)\right\}$. Hence, it is sufficient to prove that for any $\Lambda \in \sigma_{P}\left(\Gamma_{\Lambda}\right) \backslash\left\{\Lambda_{0}\right\}, \Lambda_{0}>|\Lambda|$.

For any $\Lambda \in \sigma_{P}\left(\Gamma_{\Lambda}\right)$, there is an eigenfunction $\varphi_{\Lambda}$ such that $\Gamma_{\Lambda} \varphi_{\Lambda}=\varphi_{\Lambda}$. Then we have $\left|\varphi_{\Lambda}\right|=\left|\Gamma_{\Lambda} \varphi_{\Lambda}\right| \leq \Gamma_{\operatorname{Re} \Lambda}\left|\varphi_{\Lambda}\right|$. Let $f_{\operatorname{Re} \Lambda}$ be a positive eigenfunctional corresponding to the eigenvalue $r\left(\Gamma_{\operatorname{Re} \Lambda}\right)$ of $\Gamma_{\operatorname{Re} \Lambda}$. We obtain that

$$
\left\langle f_{\operatorname{Re} \Lambda}, \Gamma_{\operatorname{Re} \Lambda}\left|\varphi_{\Lambda}\right|\right\rangle=r\left(\Gamma_{\operatorname{Re} \Lambda}\right)\left\langle f_{\operatorname{Re} \Lambda},\left|\varphi_{\Lambda}\right|\right\rangle \geq\left\langle f_{\operatorname{Re} \Lambda},\left|\varphi_{\Lambda}\right|\right\rangle
$$

Hence, $r\left(\Gamma_{\operatorname{Re} \Lambda}\right) \geq 1$ and $\operatorname{Re} \Lambda \leq \Lambda_{0}$ since $r\left(\Gamma_{\Lambda}\right)$ is strictly decreasing for $\Lambda \in \mathbb{R}$ and $r\left(\Gamma_{\Lambda}\right)=1$. If $\operatorname{Re} \Lambda=\Lambda_{0}$, then $\Gamma_{\Lambda_{0}}\left|\varphi_{\Lambda}\right|=\left|\varphi_{\Lambda}\right|$. In fact, if $\Gamma_{\Lambda_{0}}\left|\varphi_{\Lambda}\right|>\left|\varphi_{\Lambda}\right|$, taking duality pairing with the eigenfunctional $f_{\Lambda_{0}}$ corresponding to the eigenvalue $r\left(\Gamma_{\Lambda_{0}}\right)=1$ on both sides yields $\left.\left\langle f_{\Lambda_{0}}, \Gamma_{\Lambda_{0}}\left|\varphi_{\Lambda}\right|\right\rangle=\left\langle f_{\Lambda_{0}},\left|\varphi_{\Lambda}\right|\right\rangle\right\rangle\left\langle f_{\Lambda_{0}},\left|\varphi_{\Lambda}\right|\right\rangle$, which is a contradiction. Then we can write that $\left|\varphi_{\Lambda}\right|=c \varphi_{0}$, where $\varphi_{0}$ is the eigenfunction corresponding to the eigenvalue $r\left(\Gamma_{\Lambda_{0}}\right)=1$. Without loss of generality, we assume that $c=1$ and write $\varphi_{\Lambda}(a, x)=\varphi_{0}(a, x) e^{i \alpha(a, x)}$ for some real function $\alpha(a, x)$. Substituting this relation into

$$
\Gamma_{\Lambda_{0}} \varphi_{0}=\varphi_{0}=\left|\varphi_{\Lambda}\right|=\left|\Gamma_{\Lambda} \varphi_{\Lambda}\right|
$$


we have

$$
\begin{aligned}
& \int_{0}^{a^{+}} \int_{\Omega} k(a, \sigma, x, y) \int_{0}^{\sigma} e^{-\Lambda_{0}(\sigma-\tau)}\left(\mathcal{F}_{\lambda^{*}}(\tau, \sigma)\left(\varphi_{0}\left(P^{*}-I^{*}\right)\right)(\tau)\right)(y) d \tau d y d \sigma \\
& =\mid \int_{0}^{a^{+}} \int_{\Omega} k(a, \sigma, x, y) \int_{0}^{\sigma} e^{-\Lambda_{0}(\sigma-\tau)} e^{-i \operatorname{Im} \Lambda(\sigma-\tau)} \\
& \quad \times\left(\mathcal{F}_{\lambda^{*}}(\tau, \sigma)\left(\varphi_{0}\left(P^{*}-I^{*}\right)\right)(\tau)\right)(y) e^{i \alpha(\tau, y)} d \tau d y d \sigma \mid .
\end{aligned}
$$

From Heijmans (1986, Lemma 6.12), we obtain that $-\operatorname{Im} \Lambda(\sigma-\tau)+\alpha(\tau, y)=\theta$ for some constant $\theta$. By $\Gamma_{\Lambda} \varphi_{\Lambda}=\varphi_{\Lambda}$, we have $e^{i \theta} \Gamma_{\Lambda_{0}} \varphi_{0}=\varphi_{0} e^{i \alpha}$, so $\theta=\alpha(a, x)$, which implies that $\operatorname{Im} \Lambda=0$. Then, there is no element $\Lambda \in \sigma_{P}\left(\Gamma_{\Lambda}\right)$ such that $\operatorname{Re} \Lambda=\Lambda_{0}$ and $\Lambda \neq \Lambda_{0}$. Therefore, the result is proved.

Remark 7.2 Note that $\Gamma_{0} \leq M$ in the sense of positive operators since $\mathcal{F}_{\lambda^{*}} \leq \mathcal{F}_{0}$ and that when $I^{*} \equiv 0, \Gamma_{0}=M$. It follows that if $r(M)<1$, there exists only the trivial steady state $\left(P^{*}, 0\right)$ for $(1.1)$ which is locally stable. Further, if $r\left(\Gamma_{0}\right)<1<r(M)$, there exists a unique nontrivial steady state $\left(S^{*}, I^{*}\right)$ for $(1.1)$ which is locally stable. Otherwise, if $r\left(\Gamma_{0}\right)>1$, there exists a unique nontrivial steady state $\left(S^{*}, I^{*}\right)$ for $(1.1)$ which is locally unstable.

\subsection{Effects of small diffusion}

Now let us study the effects of small diffusion rate $d$ on the principal eigenvalue $\Lambda_{0}$. We write $I_{d}^{*}, \Lambda_{0}^{d}, \Gamma_{\Lambda}^{d}$ and $\mathcal{F}_{\lambda^{*}}^{d}(\tau, \sigma)$ for $I^{*}, \Lambda_{0}, \Gamma_{\Lambda}$ and $\mathcal{F}_{\lambda^{*}}(\tau, \sigma)$, respectively, to highlight the dependence on $d$.

Theorem 7.3 Let Assumption 2.2 be satisfied. Then we have

$$
\Lambda_{0}^{d} \rightarrow \Lambda_{0}^{0} \quad \text { as } \quad d \rightarrow 0
$$

where $\Lambda_{0}^{0}$ satisfies $r\left(H_{\Lambda_{0}^{0}}\right)=1$ and $H_{\Lambda}$ is defined in (7.8).

Proof Consider the following eigenvalue problem

$$
\begin{cases}\partial_{a} \psi(a, x)=-\Lambda \psi(a, x)-\left(\lambda^{*}(a, x)+\gamma(a, x)+\mu(a, x)\right) \psi(a, x)+[G \psi](a, x), & a \in\left(0, a^{+}\right], x \in \bar{\Omega}, \\ \psi(0, x)=0, & x \in \bar{\Omega}, \\ \lambda(a, x \mid \psi)=\int_{0}^{a^{+}} \int_{\Omega} k(a, \sigma, x, y) \psi(\sigma, y) d y d \sigma, & a \in\left[0, a^{+}\right], x \in \bar{\Omega}\end{cases}
$$

Solving the first equation of (7.7), we obtain

$$
\psi(a, x)=\int_{0}^{a} e^{-\Lambda(a-\sigma)} e^{-\int_{\sigma}^{a}\left(\lambda^{*}(\eta, x)+\gamma(\eta, x)+\mu(\eta, x)\right) d \eta} \lambda(\sigma, x \mid \psi)\left(P^{*}-I^{*}\right)(\sigma, x) d \sigma .
$$


Plugging it into the equation of $\lambda$, we have $\lambda(a, x)=\int_{0}^{a^{+}} \int_{\Omega} k(a, \sigma, x, y) \int_{0}^{\sigma} e^{-\Lambda(\sigma-\tau)} \Pi_{\lambda^{*}}(\tau, \sigma, y) \lambda(\tau, y)\left(P^{*}-I^{*}\right)(\tau, y) d \tau d y d \sigma$, where $\Pi_{\lambda}(\tau, \sigma, x):=e^{-\int_{\tau}^{\sigma}(\lambda(\eta, x)+\gamma(\eta, x)+\mu(\eta, x)) d \eta}$. Define an operator $H_{\Lambda}: X \rightarrow X$ by

$$
\begin{aligned}
H_{\Lambda} \varphi:= & \int_{0}^{a^{+}} \int_{\Omega} k(\cdot, \sigma, \cdot, y) \int_{0}^{\sigma} e^{-\Lambda(\sigma-\tau)} \Pi_{\lambda^{*}}(\tau, \sigma, y) \varphi(\tau, y) \\
& \times\left(P^{*}-I^{*}\right)(\tau, y) d \tau d y d \sigma, \quad \varphi \in X .
\end{aligned}
$$

By using a similar argument as in Lemma 4.1, we have that $H_{\Lambda}$ is compact and nonsupporting for every $\Lambda \in \mathbb{R}$. Hence, $\Lambda \rightarrow r\left(H_{\Lambda}\right): \mathbb{R} \rightarrow(0, \infty)$ is strictly decreasing with $\lim _{\Lambda \rightarrow-\infty} r\left(H_{\Lambda}\right)=\infty$ and $\lim _{\Lambda \rightarrow \infty} r\left(H_{\Lambda}\right)=0$. Thus there exists a unique $\Lambda_{0}^{0}$ such that $r\left(H_{\Lambda_{0}^{0}}\right)=1$. Moreover, $\Lambda_{0}^{0}$ is the principal eigenvalue of (7.7) by the argument in Theorem 7.1.

Now since $\mathcal{F}_{\lambda^{*}}^{d}(\tau, \sigma)$ is continuous with respect to $d$ in the sense of operators and $\mathcal{F}_{\lambda^{*}}^{d}(\tau, \sigma)$ converges to $\Pi_{\lambda^{*}}(\tau, \sigma, x)$ as $d \rightarrow 0, \Gamma_{\Lambda}^{d}$ converges to $H_{\Lambda}$ as $d \rightarrow 0$. Thus, by Krein-Rutman theorem, we have $r\left(\Gamma_{\Lambda}^{d}\right) \rightarrow r\left(H_{\Lambda}\right)$ as $d \rightarrow 0$, see Degla (2008, Proposition 2.1). Hence the result is concluded by the continuity of $d \rightarrow r\left(\Gamma_{\Lambda}^{d}\right)$.

Corollary 7.4 Let Assumption 2.2 be satisfied. Then the nontrivial steady state $I_{d}^{*}$ is locally stable for each $0<d \ll 1$ if $\Lambda_{0}^{0}<0$.

Proof The proof follows from Theorems 7.1 and 7.3.

Remark 7.5 We would like to mention that it is not easy in general to obtain the monotonicity of $\Lambda_{0}^{d}$ with respect to $d$. However, in some specific cases, for example if the transmission rate $k$ and birth rate $\beta$ depend only on age, and the recovery rate $\gamma$ and mortality rate $\mu$ are separated (i.e. $\gamma(a, x)=\gamma_{1}(a)+\gamma_{2}(x)$ and $\mu(a, x)=$ $\left.\mu_{1}(a)+\mu_{2}(x)\right)$ and $I^{*} \equiv 0$, then $d \rightarrow \Lambda_{0}^{d}$ is strictly decreasing. Moreover, one can prove that $\Lambda_{0}^{d} \rightarrow-\infty$ as $d \rightarrow \infty$ under the above assumptions. We will study these in details in our forthcoming papers.

At the end of this section, we investigate the asymptotic behavior of the steady state $I_{d}^{*}$ in terms of the diffusion rate. Once we know $I_{d}^{*}$, then $S^{*}$ can be easily obtained via $S^{*}=P^{*}-I_{d}^{*}$.

Theorem 7.6 Let Assumption 2.2 be satisfied. If $r(\Xi)>1$, then $I_{d}^{*} \rightarrow \tilde{I}$ as $d \rightarrow 0$, where $\tilde{I} \not \equiv 0$ satisfies the following equation

$$
\begin{cases}\partial_{a} I(a, x)=\lambda(a, x)\left(P^{*}(a, x)-I(a, x)\right)-(\gamma(a, x)+\mu(a, x)) I(a, x), & a \in\left(0, a^{+}\right], x \in \bar{\Omega} \\ I(0, x)=0, & x \in \bar{\Omega}, \\ \lambda(a, x)=\int_{0}^{a^{+}} \int_{\Omega} k(a, \sigma, x, y) I(\sigma, y) d y d \sigma, & a \in\left(0, a^{+}\right], x \in \bar{\Omega}\end{cases}
$$

and $\Xi: X \rightarrow X$ is a linear and bounded operator defined in (7.12). 
Proof Solving the first equation of (7.9), we obtain

$$
I(a, x)=\int_{0}^{a} \lambda(\sigma, x) P^{*}(\sigma, x) e^{-\int_{\sigma}^{a}(\lambda(\rho, x)+\gamma(\rho, x)+\mu(\rho, x)) d \rho} d \sigma
$$

Plugging it into the equation of $\lambda$, we have

$$
\begin{aligned}
\lambda(a, x)= & \int_{0}^{a^{+}} \int_{\Omega} k(a, \sigma, x, y) \int_{0}^{\sigma} \lambda(\tau, y) \\
& \times P^{*}(\tau, y) e^{-\int_{\tau}^{\sigma}(\lambda(\rho, y)+\gamma(\rho, y)+\mu(\rho, y)) d \rho} d \tau d y d \sigma .
\end{aligned}
$$

Define a nonlinear operator $\Psi$ on $X$ by

$$
\begin{aligned}
{[\Psi \phi](a, x):=} & \int_{0}^{a^{+}} \int_{\Omega} k(a, \sigma, x, y) \int_{0}^{\sigma} \phi(\tau, y) \\
& \times P^{*}(\tau, y) e^{-\int_{\tau}^{\sigma}(\phi(\rho, y)+\gamma(\rho, y)+\mu(\rho, y)) d \rho} d \tau d y d \sigma, \quad \phi \in X,
\end{aligned}
$$

and investigate the existence of a nontrivial positive fixed point $\phi^{*} \in X_{+} \backslash\{0\}$ such that $\Psi\left(\phi^{*}\right)=\phi^{*}$, which is no other than the desired nontrivial positive solution $\lambda \in X_{+} \backslash\{0\}$ of (7.10).

One can check that $\Psi$ is bounded and positive. Next, consider the following linear integral operator $\Xi$ on $X$ :

$$
\begin{aligned}
{[\Xi \phi](a, x):=} & \int_{0}^{a^{+}} \int_{\Omega} k(a, \sigma, x, y) \int_{0}^{\sigma} \phi(\tau, y) \\
& \times P^{*}(\tau, y) e^{-\int_{\tau}^{\sigma}(\gamma(\rho, y)+\mu(\rho, y)) d \rho} d \tau d y d \sigma, \quad \phi \in X .
\end{aligned}
$$

We can see that $\Xi$ is the Fréchert derivative of $\Psi$ at $\phi=0$ and a positive linear majorant of $\Psi$. We show that the spectral radius $r(\Xi)$ of operator $\Xi$ is a threshold value for the existence of a nontrivial fixed point of operator $\Psi$. Then by using similar arguments as in Lemma 4.1 and Proposition 4.2, we obtain that if $r(\Xi)>1$, then $\Psi$ has a positive nontrivial fixed point; that is, (7.9) has a nontrivial solution $\tilde{I}$ in $E$. Moreover, by using similar arguments as in Sect. 6 or in Busenberg et al. (1991, Theorem 4.5), one can show that $\tilde{I}$ is unique.

Next we prove the limit result. Since $\mathcal{F}_{\lambda}^{d}(\tau, \sigma)$ is continuous with respect to $d$ in the sense of operators and $\mathcal{F}_{\lambda}^{d}(\tau, \sigma)$ converges to $\Pi_{\lambda}(\tau, \sigma, x)$ as $d \rightarrow 0$, it follows that $\Phi$ converges to $\Psi$ as $d \rightarrow 0$, where $\Phi$ is defined in (4.7) and $\mathcal{F}_{\lambda}^{d}$ is defined in Lemma 2.1 with $f$ being replaced by $\lambda+\gamma+\mu$. Thus by the uniqueness of a nontrivial fixed point, we have a unique nontrivial fixed point of $\Phi$ converging to the unique one of $\Psi$ as $d \rightarrow 0$; that is $I_{d}^{*} \rightarrow \tilde{I}$. 


\section{Global stability}

In this section we discuss the global stability of system (3.5). First we introduce a condition as follows

$$
\int_{t}^{a^{+}} \int_{\Omega} k_{2}(a, y)\left(\mathcal{F}(a-t, a) I_{0}(a-t)\right)(y) d y d a>0 \text { for some } t \geq 0 .
$$

If this condition is satisfied, we say that $I_{0}$, with support in $\left[0, a^{+}\right] \times \bar{\Omega}$, is a nontrivial initial data.

Theorem 8.1 Assume that no nontrivial steady state exists, then

$$
\forall I_{0} \in E, \quad \mathcal{S}(t) I_{0} \rightarrow 0 \text { as } t \rightarrow \infty
$$

The proof is identical to that of Theorem 5.1 in Busenberg et al. (1991). So we omit it.

Proposition 8.2 Let Assumption 2.2 be satisfied and $I_{0}$ be a nontrivial initial data. Then there exist $t_{0}>0$ and $\xi>0$ such that

$$
\mathcal{S}\left(t_{0}\right) I_{0} \geq \xi I^{*}
$$

Proof The proof is inspired by Proposition 5.2 in Busenberg et al. (1991). Noting (3.15), we have

$$
I(t) \geq \frac{1}{\alpha} \int_{0}^{t} e^{-\frac{1}{\alpha}(t-s)} e^{(t-s) A}[I(s)+\alpha F(I(s))] d s .
$$

Now since $\alpha<\frac{1}{\left(\lambda^{+}+\gamma^{+}\right)}$, we have

$$
I(s)+\alpha F(I(s)) \geq w(s),
$$

where

$$
w(s)(a, x)=\alpha \epsilon k_{1}(a, x) P^{*}(a, x) \int_{0}^{a^{+}} \int_{\Omega} k_{2}(\sigma, y) I(s)(\sigma, y) d y d \sigma,
$$

so that

$$
\begin{aligned}
& e^{(t-s) A} w(s)(a, x) \\
& \geq \begin{cases}\alpha \in\left(\mathcal{F}(a+s-t, a)\left(k_{1} P^{*}\right)(a+s-t)\right)(x) \int_{0}^{a^{+}} \int_{\Omega} k_{2}(\sigma, y) I(s)(\sigma, y) d y d \sigma & \text { for } a>t-s, \\
0 & \text { for } a<t-s .\end{cases}
\end{aligned}
$$

Plugging it into (8.2), we obtain

$$
I(t)(a, x) \geq \epsilon e^{-\frac{1}{\alpha} t} \int_{(t-a) \vee 0}^{t}\left(\mathcal{F}(a+s-t, a)\left(k_{1} P^{*}\right)(a+s-t)\right)(x) h(s) d s
$$


with

$$
h(s)=\int_{0}^{a^{+}} \int_{\Omega} k_{2}(\sigma, y) I(s)(\sigma, y) d y d \sigma .
$$

We make the following claim.

Claim 8.3 There exists $s_{\infty}$ such that $h(s)>0$ for $s>s_{\infty}$.

To prove this claim, we first prove another claim.

Claim 8.4 If $h(s)>0$ for $s \in\left[s_{1}, s_{2}\right]$, then $h(s)>0$ for $s \in\left[s_{1}-a^{+}, s_{2}+a^{+}\right]$.

Note first that $I(t)(a, x)>0$ for all $x \in \bar{\Omega}$ if

$$
a>0, \quad t>s_{1}, \quad s_{1}-a^{+}<t-a<s_{2} .
$$

In fact, if these are satisfied, then $(t-a) \vee s_{1}<t \wedge s_{2}$. Setting $I=\left[(t-a) \vee s_{1}, t \wedge s_{2}\right]$, we have

$$
I \subset\left[s_{1}, s_{2}\right], \quad\{a-t+I\} \cap\left[0, a^{+}\right] \neq \varnothing,
$$

where $\{a-t+I\}:=\{a-t+i: i \in I\}$. So it follows from (8.3) that

$$
\begin{aligned}
I(t)(a, x) \geq & \epsilon e^{-\frac{1}{\alpha} t} \int_{(t-a) \vee s_{1}}^{t \wedge s_{2}}\left(\mathcal{F}(a+s-t, a)\left(k_{1} P^{*}\right)(a+s-t)\right)(x) \\
& \times h(s) d s>0 \text { for all } x \in \bar{\Omega},
\end{aligned}
$$

because by (8.5) the integrand is positive on some interval contained in $I$.

Finally, fixing $t \in\left[s_{1}-a^{+}, s_{2}+a^{+}\right]$, noting that $k_{2}$ is positive on $\left[0, a^{+}\right] \times \bar{\Omega}$, and using (8.4), we know that $I(t)(a, x)$ is positive for $a>0$ and $a \in R:=\left[t-s_{2}, t+\right.$ $\left.a^{+}-s_{1}\right]$. But our choice of $t$ yields

$$
t-s_{2}<a^{+}, \quad t+a^{+}-s_{1}>a^{+}-a^{+}=0
$$

so that $R \cap\left[0, a^{+}\right] \neq \varnothing$ and $h(t)>0$.

Now Claim 8.4 is proven. Note that if $I_{0}$ is nontrivial, then from (3.15) we see that for some positive constant $\delta$,

$$
\begin{aligned}
h(s) & =\int_{0}^{a^{+}} \int_{\Omega} k_{2}(\sigma, y) I(s)(\sigma, y) d y d \sigma \geq \delta \int_{0}^{a^{+}} \int_{\Omega} k_{2}(\sigma, y)\left(e^{s A} I_{0}\right)(\sigma, y) d y d \sigma \\
& =\delta \int_{s}^{a^{+}} \int_{\Omega} k_{2}(\sigma, y)\left(\mathcal{F}(\sigma-s, \sigma) I_{0}(\sigma-s)\right)(y) d y d \sigma>0
\end{aligned}
$$

for $s$ in some interval $\left[s_{1}, s_{2}\right]$. Thus, iterating Claim 8.4 we have for any integer $n$ that

$$
h(s)>0 \text { in }\left[s_{1}-n a^{+}, s_{2}+n a^{+}\right] .
$$


So if $n$ is sufficiently large, successive intervals are overlapping, and consequently Claim 8.3 is desired.

We now use Claim 8.3 to the prove following result.

Claim 8.5 There exists $t_{\infty}$ such that $I(t)(a, x)>0$ for $a>0, x \in \bar{\Omega}$ and $t>t_{\infty}$.

In fact, if $t>s_{\infty}+2 a^{+}$, then $t-a>s_{\infty}$. By (8.3), we have

$$
\begin{aligned}
I(t)(a, x) & \geq \epsilon e^{-\frac{1}{\alpha} t} \int_{t-a}^{t}\left(\mathcal{F}(a+s-t, a)\left(k_{1} P^{*}\right)(a+s-t)\right)(x) h(s) d s \\
& =\epsilon e^{-\frac{1}{\alpha} t} \int_{0}^{a}\left(\mathcal{F}(\sigma, a)\left(k_{1} P^{*}\right)(\sigma)\right)(x) h(\sigma+t-a) d \sigma>0 .
\end{aligned}
$$

Now let $t_{0}>s_{\infty}+a^{+}$. Then $t_{0}-a>s_{\infty}$ and

$$
\begin{aligned}
I\left(t_{0}\right)(a, x) & \geq \epsilon e^{-\frac{1}{\alpha} t_{0}} \int_{t_{0}-a}^{t_{0}}\left(\mathcal{F}\left(a+s-t_{0}, a\right)\left(k_{1} P^{*}\right)\left(a+s-t_{0}\right)\right)(x) h(s) d s \\
& >\epsilon e^{-\frac{1}{\alpha} t_{0}} h_{-} \int_{t_{0}-a}^{t_{0}}\left(\mathcal{F}\left(a+s-t_{0}, a\right)\left(k_{1} P^{*}\right)\left(a+s-t_{0}\right)\right)(x) d s \\
& =\epsilon e^{-\frac{1}{\alpha} t_{0}} h_{-} \int_{0}^{a}\left(\mathcal{F}(s, a)\left(k_{1} P^{*}\right)(s)\right)(x) d s=\epsilon e^{-\frac{1}{\alpha} t_{0}} h_{-} m(a, x)(8.7
\end{aligned}
$$

where $h_{-}:=\inf _{s \in\left[s_{\infty}, t_{0}\right]} h(s)$. Finally, by (6.4) we have

$$
I\left(t_{0}\right)(a, x) \geq \xi I^{*}(a, x),
$$

where $\xi=\frac{\epsilon}{\epsilon_{2}} e^{-\frac{1}{\alpha} t_{0}} h_{-}$and the proof is complete.

Theorem 8.6 Let Assumption 2.2 be satisfied. Let $I^{*}$ be the unique nontrivial steady state. Then for any nontrivial initial data $I_{0}$ we have

$$
\mathcal{S}(t) I_{0} \rightarrow I^{*} \text { as } t \rightarrow \infty
$$

If $I_{0}$ is not nontrivial, then

$$
\mathcal{S}(t) I_{0}=0 \text { for } t \geq a^{+}
$$

Proof Inspired by Theorem 5.3 in Busenberg et al. (1991), let $\xi$ and $t_{0}$ be as in previous proposition. We have

$$
\xi I^{*} \leq \mathcal{S}\left(t_{0}\right) I_{0} \leq P^{*}
$$

and

$$
\mathcal{S}(t) \xi I^{*} \leq \mathcal{S}\left(t+t_{0}\right) I_{0} \leq \mathcal{S}(t) P^{*}
$$


Now

$$
\xi I^{*}=\xi \mathcal{S}(t) I^{*} \leq \mathcal{S}(t) \xi I^{*}, \quad \mathcal{S}(t) P^{*} \leq P^{*}
$$

Consequently, $\mathcal{S}(t) \xi I^{*}$ and $\mathcal{S}(t) P^{*}$ are respectively nondecreasing and nonincreasing with respect to $t$ and converge to a nontrivial steady state which coincides with $I^{*}$ by the uniqueness Theorem 6.5. Then the first result (8.8) follows from (8.10).

Next let

$$
\int_{t}^{a^{+}} \int_{\Omega} k_{2}(\sigma, y)\left(\mathcal{F}(\sigma-t, \sigma) I_{0}(\sigma-t)\right)(y) d y d \sigma=0 \text { for all } t \geq 0 .
$$

Let us show that the iterates $I^{n}(t)$ defined in (3.16) satisfy

$$
\int_{s}^{a^{+}} \int_{\Omega} k_{2}(a, y) I^{n}(t)(a-s, y) d y d a=0 \text { for all } t \geq 0, s \geq 0 .
$$

In fact, if (8.11) is true, then

$$
\lambda\left[a, x \mid I^{n}(t)(\cdot, \cdot)\right] \leq k_{1}(a, x) \int_{0}^{a^{+}} \int_{\Omega} k_{2}(\sigma, y) I^{n}(t)(\sigma, y) d y d \sigma=0
$$

and

$$
I^{n}(t)+\alpha F\left(I^{n}(t)\right) \leq I^{n}(t)
$$

This implies that

$$
I^{n+1}(t) \leq e^{t A} I_{0}+c \int_{0}^{t} e^{(t-\sigma) A} I^{n}(\sigma) d \sigma
$$

where $c>0$ and consequently,

$$
\begin{aligned}
& \int_{s}^{a^{+}} \int_{\Omega} k_{2}(\sigma, y) I^{n+1}(t)(\sigma-s, y) d y d \sigma \\
& \leq \int_{s+t}^{a^{+}} \int_{\Omega} k_{2}(\sigma, y)\left(\mathcal{F}(\sigma-t-s, \sigma-s) I_{0}(\sigma-t-s)\right)(y) d y d \sigma \\
& \quad+c \int_{0}^{t} \int_{s+t-\eta}^{a^{+}} \int_{\Omega} k_{2}(\sigma, y)(\mathcal{F}(\sigma-t-s+\eta, \sigma-s) \\
& \left.\quad \times I^{n}(\eta)(\sigma-t-s+\eta)\right)(y) d y d \sigma d \eta=0 .
\end{aligned}
$$


Thus, (8.11) is true by induction, where we used the positivity of $k_{2}$ and strong positivity of semigroup $\mathcal{F}(\sigma, a)$ for $a-\sigma \geq 0$,

$$
\begin{aligned}
& \int_{s+t-\eta}^{a^{+}} \int_{\Omega} k_{2}(\sigma, y)\left(\mathcal{F}(\sigma-t-s+\eta, \sigma-s) I^{n}(\eta)(\sigma-t-s+\eta)\right)(y) d y d \sigma=0, \\
& \forall \eta \geq 0 .
\end{aligned}
$$

Finally (8.11) implies that

$$
\int_{s}^{a^{+}} \int_{\Omega} k_{2}(a, y) I(t)(a-s, y) d y d a=0 \text { for all } t \geq 0, s \geq 0 .
$$

So if $t>a^{+}$, then

$$
I(t) \leq c \int_{0}^{t} e^{(t-s) A} I(s) d s \text { in }\left[0, a^{+}\right] \times \bar{\Omega},
$$

which implies the second result (8.9), see the argument in Theorem 5.3 in Busenberg et al. (1991).

\section{Discussion}

In this paper, we studied an age-structured epidemic model with nonlocal (convolution) diffusion which describes the spatial spread (via long-distance travel) of an infectious disease in a host population with age structure. We first analyzed the well-posedness of the model, then investigated the existence and uniqueness of the nontrivial steady state corresponding to an endemic state, and finally studied the local and global stability of this nontrivial steady state. Moreover, we considered the asymptotic properties of the principal eigenvalue and nontrivial steady state with respect to the diffusion rate.

Recall that the basic reproduction number is defined by the spectral radius of the next generation operator, $\mathcal{R}_{0}:=r(\mathcal{G})$, where $\mathcal{G}$ is given by (5.3). We would like to point out that the effects of age and nonlocal diffusion were implicitly included in the expression of the operator $\mathcal{G}$. Theorem 5.2 stated that $\mathcal{R}_{0}$ is a threshold for the existence of a nontrivial steady state; namely, system (1.1) has only the trivial steady state $\left(P^{*}, 0\right)$ if $\mathcal{R}_{0} \leq 1$ and has at least one nontrivial steady state $\left(S^{*}, I^{*}\right)$ if $\mathcal{R}_{0}>1$. For the operator $\Gamma_{\Lambda}$ defined by (7.6), which also depends on age and nonlocal diffusion implicitly, there exists a unique (principal) eigenvalue $\Lambda_{0}$ such that $r\left(\Gamma_{\Lambda_{0}}\right)=1$. Thus, Theorem 7.1 indicates that $r\left(\Gamma_{0}\right)$ plays as another threshold for the stability of the nontrivial steady state; namely, it is locally stable when $r\left(\Gamma_{0}\right)<1$ and unstable when $r\left(\Gamma_{0}\right)>1$. Rewrite the principal eigenvalue as $\Lambda_{0}^{d}$ to represent the fact that it depends on the diffusion rate $d$. If the transmission rate $k$, birth rate $\beta$ depend only on age and the recovery rate $\gamma$, mortality rate $\mu$ are separated and $I^{*} \equiv 0$, then $d \rightarrow \Lambda_{0}^{d}$ is strictly decreasing. Moreover, $\Lambda_{0}^{d} \rightarrow-\infty$ as $d \rightarrow \infty$.

We would like to mention again that the analysis of (1.1) with nonlocal diffusion is also valid for (1.6) with Laplace diffusion; that is, $\mathcal{L} u:=\Delta u$, see Kuniya and Oizumi 
(2015) for the existence of steady states for an age-structured SIS epidemic model with Laplace diffusion. It is also interesting to investigate the principal eigenvalue of the age-structured operator coupled with more complicated nonlocal diffusion operator in SIS epidemic models (for example including cost parameter and diffusion range in our forthcoming paper), and use it as a threshold to study the dynamics behavior of SIS epidemic models, see Yang et al. (2019). Note that we assumed $d_{I}=d_{S}$ in (1.1), it is also valuable to consider different diffusion rates, for example, $d_{I} \neq d_{S}$. In addition, the method of monotone and positive operators will not be valid if we consider more complicated epidemic models, for example SIR or SEIR age-structured models with nonlocal diffusion (which might be suitable to describe the geographic spread of COVID-19), since the system will lose monotonicity. Thus, it is necessary to study such more complicated epidemic models by using a different approach. We leave these for future consideration.

Acknowledgements We are very grateful to the anonymous reviewers for their helpful comments and constructive suggestions which helped us to improve the presentation of the paper.

\section{References}

Blyuss KB (2005) On a model of spatial spread of epidemics with long-distance travel. Phys Lett A 345(13):129-136

Brezis H (2010) Functional analysis. Sobolev Spaces and Partial Differential Equations. Springer Science and Business Media, New York

Busenberg SN, Iannelli M, Thieme HR (1991) Global behavior of an age-structured epidemic model. SIAM J Math Anal 22(4):1065-1080

Chekroun A, Kuniya T (2020) Global threshold dynamics of an infection age-structured SIR epidemic model with diffusion under the dirichlet boundary condition. J Differ Equ 269(8):117-148

Degla G (2008) An overview of semi-continuity results on the spectral radius and positivity. J Math Anal Appl 338(1):101-110

Di Blasio G (2010) Mathematical analysis for an epidemic model with spatial and age structure. J Evol Equ 10(4):929-953

Diekmann O, Heesterbeek JAP, Metz JAJ (1990) On the definition and the computation of the basic reproduction ratio $R_{0}$ in models for infectious diseases in heterogeneous populations. J Math Biol 28(4):365-382

Findlater A, Bogoch II (2018) Human mobility and the global spread of infectious diseases: a focus on air travel. Trends Parasitol 34(9):772-783

Fitzgibbon WE, Parrott ME, Webb GF (1994) Diffusive epidemic models with spatial and age dependent heterogeneity. Discrete Contin Dyn Syst 1(1):35-37

Fitzgibbon WE, Parrott ME, Webb GF (1996) A diffusive age-structured SEIRS epidemic model. Methods Appl Anal 3(3):358-369

Guo BZ, Chan WL (1994) On the semigroup for age dependent population dynamics with spatial diffusion. J Math Anal Appl 184(1):190-199

Heijmans HJ (1986) The dynamical behaviour of the age-size-distribution of a cell population. The dynamics of physiologically structured populations. Springer, New York, pp 185-202

Iannelli M (1995) Mathematical theory of age-structured population dynamics. Giardini editori e stampatori, Pisa

Iannelli M, Milner FA (2017) The basic approach to age-structured population dynamics. Springer, New York

Iannelli M, Milner FA, Pugliese A (1992) Analytical and numerical results for the age-structured SIS epidemic model with mixed inter-intracohort transmission. SIAM J Math Anal 23(3):662-688

Iannelli M, Kim MY, Park EJ (1999) Asymptotic behavior for an SIS epidemic model and its approximation. Nonlinear Anal 35(7):797-814 
Inaba H (1990) Threshold and stability results for an age-structured epidemic model. J Math Biol 28(4):411434

Inaba H (2014) On a pandemic threshold theorem of the early Kermack-McKendrick model with individual heterogeneity. Math Popul Stud 21(2):95-111

Inaba H (2017) Age-structured population dynamics in demography and epidemiology. Springer, New York

Kang H, Ruan S (2021a) Approximation of random diffusion by nonlocal diffusion in age-structured models. Z Angew Math Phys 72:78

Kang H, Ruan S (2021b) Nonlinear age-structured population models with nonlocal diffusion and nonlocal boundary conditions. J Differ Equ 278:430-462

Kang H, Huang Q, Ruan S (2020a) Periodic solutions of an age-structured epidemic model with periodic infection rate. Commun Pure Appl Anal 19(10):4955-4972

Kang H, Ruan S, Yu X (2020b) Age-structured population dynamics with nonlocal diffusion. J Dyn Differ Equ. https://doi.org/10.1007/s10884-020-09860-5

Kim MY (2006) Global dynamics of approximate solutions to an age-structured epidemic model with diffusion. Adv Comput Math 25(4):451-474

Kubo M, Langlais M (1994) Periodic solutions for nonlinear population dynamics models with agedependence and spatial structure. J Differ Equ 109(2):274-294

Kuniya T (2014) Existence of a nontrivial periodic solution in an age-structured SIR epidemic model with time periodic coefficients. Appl Math Lett 27:15-20

Kuniya T, Iannelli M (2014) $R_{0}$ and the global behavior of an age-structured SIS epidemic model with periodicity and vertical transmission. Math Biosci Eng 11(4):929-945

Kuniya T, Inaba H (2013) Endemic threshold results for an age-structured SIS epidemic model with periodic parameters. J Math Anal Appl 402(2):477-492

Kuniya T, Oizumi R (2015) Existence result for an age-structured SIS epidemic model with spatial diffusion. Nonlinear Anal Real World Appl 23:196-208

Kuniya T, Wang J, Inaba H (2016) A multi-group SIR epidemic model with age structure. Discrete Contin Dyn Syst B 21(10):3515

Kuniya T, Inaba H, Yang J (2018) Global behavior of SIS epidemic models with age structure and spatial heterogeneity. Jpn J Ind Appl Math 35(2):669-706

Langlais M, Busenberg S (1997) Global behaviour in age structured SIS models with seasonal periodicities and vertical transmission. J Math Anal Appl 213(2):511-533

Marek I (1970) Frobenius theory of positive operators: comparison theorems and applications. SIAM J Appl Math 19(3):607-628

Ruan S (2007) Spatial-temporal dynamics in nonlocal epidemiological models. In: Takeuchi Y, Sato K, Iwasa Y (eds) Mathematics for life science and medicine. Springer-Verlag, Berlin, pp 97-122

Sawashima I (1964) On spectral properties of some positive operators. Nat Sci Rep Ochanomizu Univ 15(2):53-64

Thieme HR (1991) Stability change of the endemic equilibrium in age-structured models for the spread of S-I-R type infectious diseases. Differential equations models in biology. Springer Epidemiology and Ecology, New York, pp 139-158

Webb GF (1980) An age-dependent epidemic model with spatial diffusion. Arch Ration Mech Anal 75(1):91-102

Webb GF (1982) A recovery-relapse epidemic model with spatial diffusion. J Math Biol 14(2):177-194

Webb GF (1984) Theory of nonlinear age-dependent population dynamics. Marcel Dekker, New York

Xu WB, Li WT, Ruan S (2020) Spatial propagation in an epidemic model with nonlocal diffusion: the influences of initial data and dispersals. Sci China Math 63(11):2177-2206

Yang FY, Li WT (2017) Dynamics of a nonlocal dispersal SIS epidemic model. Comm Pure Appl Anal 16(3):781-798

Yang FY, Li Y, Li WT, Wang ZC (2013) Traveling waves in a nonlocal dispersal Kermack-McKendrick epidemic model. Discrete Contin Dynam Syst Ser B 18(7):1969-1993

Yang FY, Li WT, Ruan S (2019) Dynamics of a nonlocal dispersal SIS epidemic model with Neumann boundary conditions. J Differ Equ 267(3):2011-2051

Zhao G, Ruan S (2018) Spatial and temporal dynamics of a nonlocal viral infection model. SIAM J Appl Math 78(4):1954-1980

Publisher's Note Springer Nature remains neutral with regard to jurisdictional claims in published maps and institutional affiliations. 